\title{
Centriole distal-end proteins CP110 and Cep97 influence cartwheel growth at the proximal-end of centrioles
}

\author{
Mustafa G. Aydogan ${ }^{1,2, *, \dagger}$, Laura E. Hankins ${ }^{1, *}$, Thomas L. Steinacker ${ }^{1}$, \\ Mohammad Mofatteh ${ }^{1}$, Saroj Saurya ${ }^{1}$, Alan Wainman ${ }^{1}$, Siu S. Wong ${ }^{1}$, Xin Lu$^{3}$, \\ Felix Y. Zhou ${ }^{3, \dagger}$ and Jordan W. Raff ${ }^{1, \dagger}$
}

\section{Affiliation:}

${ }^{1}$ Sir William Dunn School of Pathology, University of Oxford, Oxford, UK.

${ }^{2}$ Department of Biochemistry and Biophysics, University of California, San Francisco, USA.

${ }^{3}$ Ludwig Institute for Cancer Research, Nuffield Department of Clinical Medicine, University of Oxford, Oxford, UK.

*These authors contributed equally.

†Corresponding authors. E-mail: mustafa.aydogan@ucsf.edu (MGA); felix.zhou@ludwig.ox.ac.uk (FYZ); jordan.raff@path.ox.ac.uk (JWR) 


\section{Summary}

Centrioles are composed of a central cartwheel tethered to nine-fold symmetric microtubule (MT) blades. The centriole cartwheel and MTs are thought to grow from opposite ends of these organelles, so it is unclear how they coordinate their assembly. We previously showed that an oscillation of Polo-like kinase 4 (Plk4) initiates and times the growth of the cartwheel at the proximal end. Here, we show that CP110 and Cep97 form a complex close to the distal-end of the centriole MTs whose levels oscillate as the new centriole MTs grow. The CP110/Cep97 oscillation does not appear to time the period of centriole MT growth, but rather the oscillation is entrained by the core Cdk/Cyclin oscillator that drives the nuclear divisions in these embryos. We find that changing the levels of CP110/Cep97 alters the Plk4 oscillation and the growth of the cartwheel at the proximal end. These findings reveal an unexpected crosstalk between factors normally concentrated at opposite ends of the growing centrioles, which may help to coordinate centriole growth. 


\section{Introduction}

The cytoplasm is a compact environment, filled with many different types of organelles that often have structurally complex conformations. How these organelles coordinate their assembly in a precise and timely manner is a fundamental question in cell biology (Liu et al., 2018; Mukherji and O'Shea, 2014). Centrioles are cytoskeletal organelles, whose linear structure makes them an excellent model with which to study the principles of organelle biogenesis. In most dividing cells, centrioles duplicate in S-phase when a daughter centriole assembles from the side of an existing mother centriole (Arquint and Nigg, 2016; Banterle and Gönczy, 2017; Fırat-Karalar and Stearns, 2014). Newly formed centrioles are composed of an inner cartwheel and surrounding microtubule (MT) blades-two structures that are thought to be assembled at opposite ends of the growing daughter centriole (Breslow and Holland, 2019; Gemble and Basto, 2018). In many species, the cartwheel and MT blades grow to approximately the same size (Winey and O'Toole, 2014), indicating that the growth of the centriole cartwheel and centriole MTs are coordinated to ensure that these structures grow to consistent absolute and relative sizes. There is also a second phase of centriole growth that occurs largely in $G_{2}$, and in some species or cell-types this can be substantial, so that the centriole MT blades extend well beyond the cartwheel; this second phase of growth may be less tightly regulated (Kong et al., 2020).

We recently showed in early Drosophila embryos that cartwheel assembly is homeostatic: when cartwheels grow slowly, they grow for a longer period, and vice versa to maintain a constant size (Aydogan et al., 2018). Plk4 appears to 
enforce this homeostatic behaviour by forming an oscillating system at the base of the growing cartwheel that can be entrained by the core Cdk/Cyclin cell-cycle oscillator (CCO) to initiate and time cartwheel formation in S-phase (Aydogan et al., 2020). In contrast, we know relatively little about how the growth of the centriole MTs is regulated (Sharma et al., 2021). Proteins such as Sas-4/CPAP (Schmidt et al., 2009; Sharma et al., 2016; Zheng et al., 2016), Cep135/Bld10 (Dahl et al., 2015; Lin et al., 2013) and Ana1/Cep295 (Chang et al., 2016; Saurya et al., 2016) are thought to interact with the centriole MTs to promote centriole MT growth, while proteins such as CP110 and Cep97 are concentrated at the distal-end of the centrioles where they appear to suppress centriole MT growth (Delgehyr et al., 2012; Franz et al., 2013; Kohlmaier et al., 2009; Schmidt et al., 2009; Spektor et al., 2007). It is unclear, however, how these proteins are regulated to ensure that the centriole MTs grow to the correct size, nor how this growth is coordinated with the growth of the centriole cartwheel.

Here we show that in early fly embryos the levels of CP110 and Cep97 oscillate at the distal end of the growing daughter centriole during S-phase. The CP110/Cep97 oscillation does not appear to set the period of daughter centriole MT growth, but the levels of CP110 and Cep97 in the embryo can influence the growth of the centriole cartwheel, apparently by tuning the Plk4 oscillation at the base of the growing daughter. These findings reveal that crosstalk between factors normally localized to opposite ends of the growing centrioles could help to coordinate centriole cartwheel and MT growth. 


\section{Results}

\section{CP110 and Cep97 form a complex close to the $(+)$ end of centriole MTs}

To examine the centriolar localization of CP110 and Cep97 in Drosophila, we generated transgenic fly lines expressing either CP110-GFP or GFP-Cep97 under the control of the Ubiquitin (Ubq) promoter (uCP110-GFP and UGFPCep97) and Asl-mCherry (a marker for the mother centriole) (Novak et al., 2014) (Fig. 1A). 3D-Structured Illumination super-resolution Microscopy (3DSIM) in fly wing-disc cells confirmed previous observations that both proteins localize to the distal-end of the mother and daughter centrioles (Dobbelaere et al., 2020; Franz et al., 2013), and revealed that both proteins form a ring that is of the correct size to be positioned at, or very close to, the plus-ends of the centriole MTs (Fig. 1, B and C)—consistent with previous reports (Guennec et al., 2020; Yang et al., 2018). Using genetic deletions of CP110 (Franz et al., 2013) and Cep97 (Dobbelaere et al., 2020), we found that the cytoplasmic levels of each protein appeared somewhat reduced in the absence of the other, most dramatically for CP110 in the absence of Cep97 (Fig. S1A). The centriolar localisation of each protein was also dependent on the other (Fig. S1, B and C)-supporting the view that these proteins form a complex at the distal end of the centriole MTs (Delgehyr et al., 2012; Dobbelaere et al., 2020; Franz et al., 2013; Kohlmaier et al., 2009; Schmidt et al., 2009; Spektor et al., 2007).

The CP110/Cep97 complex is recruited to the growing daughter centriole in $\underline{\text { an oscillatory manner }}$ 
In the absence of the CP110/Cep97 complex the centriole MTs are dramatically elongated (Dobbelaere et al., 2020; Franz et al., 2013; Schmidt et al., 2009). It is unclear, however, if this is because the MTs on the growing daughter centrioles grow too quickly, or because the centriole MTs on the mother centriole are not properly "capped” to prevent inappropriate MT growth-or a combination of the two. To determine whether the CP110/Cep97 complex was recruited to growing daughter centrioles, we examined the dynamics of CP110/Cep97 localisation to centrioles in living fly embryos, monitoring their recruitment over nuclear cycles 11-13 (Movies S1 and S2). Unexpectedly, uCP110-GFP and uGFP-Cep97 were recruited to centrioles in an oscillatory manner, with centriolar levels being lowest during mitosis and peaking in approximately mid-S-phase (Fig. 2, A and B). The oscillations of both proteins appeared to be largely in phase with each other, and we confirmed that this was indeed the case in embryos simultaneously expressing uCP110-GFP and uRFP-Cep97 (Fig. 2C; Movie S3).

To test whether these oscillations were occurring at the mother and/or at the growing daughter centriole, we examined uCP110-GFP or uGFP-Cep97 recruitment to centrioles in embryos co-expressing Asl-mCherry using Airyscan super resolution microscopy. This analysis revealed that uCP110-GFP or uGFP-Cep97 levels remained relatively constant on mother centrioles during S-phase, but exhibited a clear cyclical behaviour on the growing daughter (Fig. 3). We conclude that the levels of CP110 and Cep97 oscillate at the tip of the growing daughter centriole during S-phase. 
These observations are in contrast with a recent report in syncytial fly embryos that Cep97-GFP expressed under its endogenous promoter is first recruited to new-born centrioles late in S-phase, after they have already fully elongated (Dobbelaere et al., 2020). We wondered, therefore, whether our observations might be an artefact of overexpression, as the Ubq-controlled proteins we used here are overexpressed by $\sim 2-5 x$ compared to their endogenous proteins (Fig. S1A). We therefore analysed the recruitment of CP110-GFP and Cep97-GFP to centrioles during nuclear cycle 12 using transgenic lines in which each protein was expressed from their endogenous promoters (eCP110-GFP and eCep97-GFP, respectively). Both fusion proteins were expressed at similar or slightly lower levels than their endogenous proteins (Fig. 4, A and B) but exhibited a clear oscillatory recruitment during S-phase (Fig. 4, C and D). The oscillation phase was similar to that seen when the proteins were expressed from the Ubq promoter, but the amplitude was greatly reduced, indicating that the endogenously expressed proteins were recruited to centrioles at lower levels than their overexpressed counterparts (Fig. 4, C and D). Thus, in our hands, CP110GFP and GFP-Cep97 are recruited to growing daughter centrioles in an oscillatory manner even when expressed at endogenous levels.

The growth period of the centriole MTs does not appear to be set by the $\underline{\text { CP110/Cep97 oscillation }}$

The CP110/Cep97 oscillation peaked in mid-S-phase (Fig. 4, C and D), which is about the same time that the daughter centrioles normally stop growing (Aydogan et al., 2018). We wondered, therefore, whether the gradual 
accumulation of CP110/Cep97 at the distal end of the growing daughter MTs might influence the time at which the centriole MTs stop growing. We noticed, however, that while overexpressing either uGFP-Cep97 or uCP110-GFP dramatically increased the amplitude of their respective oscillations when compared to eGFP-Cep97 and eCP110-GFP (judged by their peak intensity), it did not dramatically change their relative phase (judged by their absolute and S-phase normalized peak centres) (Fig. 4, E and F). Inspection of these oscillations revealed that the centriolar levels of uGFP-Cep97 and uCP110GFP were already higher at the start of S-phase than the peak levels attained in the eGFP-Cep97 and eCP110-GFP embryos (Fig. 4, C and D). Thus, it seems unlikely that the centriole MTs stop growing when CP110/Cep97 levels reach a threshold level at the distal end, as the centriole MTs would hardly grow at all in S-phase in the uGFP-Cep97 and uCP110-GFP embryos if this were the case. Indeed, previous electron microscopy studies have shown that overexpressing CP110 only slightly shortens centriole length in Drosophila wing disc cells (Franz et al., 2013).

\section{The CP110/Cep97 oscillation is entrained by the Cdk/Cyclin cell cycle}

\section{oscillator}

These overexpression studies demonstrate that centriolar CP110/Cep97 levels do not normally peak in mid-S-phase because the centriolar binding sites for the complex are saturated; significantly more CP110/Cep97 can be recruited to centrioles when these proteins are simply overexpressed (Fig. 4). We wondered, therefore whether the CP110/Cep97 oscillation might be entrained by the core Cdk/Cyclin cell cycle oscillator (CCO) that drives the 
nuclear divisions in these early embryos. During nuclear cycles 11-13, the rate of CCO activation during S-phase gradually slows, leading to the lengthening of S-phase at successive cycles (Farrell and O'Farrell, 2014; Liu et al., 2021). We noticed that there was a strong and significant correlation between the timing of the CP110/Cep97 peak and S-phase length for both uGFP-Cep97 and uCP110-GFP at all nuclear cycles (average $r=0.86 \pm 0.19$; p<0.0001 except for uGFP-Cep97 in Cycle 11, where $p=0.04$ ) (Fig. 5, A and B). This correlation was also observed in the eCep97-GFP and eCP110-GFP lines that we analysed during nuclear cycle 12 (average $r=0.86 \pm 0.08$; $p=<0.0001$ ) (Fig. 5C). Taken together, these studies suggest that the recruitment of CP110/Cep97 to centrioles is regulated by the CCO.

\section{The CP110/Cep97 complex influences the rate and period of centriole}

\section{cartwheel growth}

We next tested whether CP110/Cep97 might influence the growth of the central cartwheel. This may seem counterintuitive, as we previously showed that an oscillation in Plk4 levels stimulates cartwheel growth at the proximal end of the centriole (Aydogan et al., 2018, 2020), while we show here that CP110/Cep97 levels oscillate at the distal end of the growing daughter. Nevertheless, the ability of Plk4 to promote centriole overduplication in human cells requires CP110 (Kleylein-Sohn et al., 2007) and the phosphorylation of CP110 by Plk4 is required for efficient centriole assembly in at least some systems (Lee et al., 2017), hinting at possible crosstalk between Plk4 and CP110/Cep97. 
We previously used the incorporation of the core centriole cartwheel component Sas-6-GFP as a proxy to measure centriole cartwheel growth (Aydogan et al., 2018), so we applied this assay to determine the parameters of cartwheel growth in embryos that either lacked or overexpressed CP110 or Cep97 (Fig. S1A). Embryos with these mutations are viable and display no obvious developmental defects (Fig. S1D). As an important control, we confirmed that Sas-6-GFP was incorporated only into the proximal end of the growing daughter centrioles even in the absence of CP110 or Cep97 (Fig. S2). This excludes the possibility that Sas-6 can inappropriately incorporate into the distal-end of either the mother centrioles or the growing daughter centrioles if they are not capped by CP110 or Cep97.

To our surprise, the centriole cartwheel grew more quickly when CP110 or Cep97 were absent, and more slowly when either protein was overexpressed (Fig. 6), demonstrating that CP110/Cep97 levels in the embryo influence the rate of cartwheel growth. Cartwheel growth is normally regulated homeostatically: when cartwheels grow faster, they grow for a shorter period (and vice versa) (Aydogan et al., 2018). This homeostatic regulation appeared to be largely, but not perfectly, maintained in embryos in which the levels of CP110 or Cep97 were altered: the cartwheels grew faster, but for a shorter period in embryos lacking CP110 or Cep97, and more slowly, but for a longer period in embryos overexpressing CP110 or Cep97 (Fig. 6). The changes in the growth period, however, were not sufficient to compensate for the more dramatic changes in the growth rate, so the cartwheels were slightly longer in embryos lacking CP110 or Cep97, and slightly shorter in embryos 
overexpressing CP110 or Cep97 (Fig. 6). This is in good agreement with previous electron microscopy observations that the core centriole structure in wing disc cells lacking CP110 is slightly longer (although the centriole MTs are dramatically elongated), while in cells overexpressing CP110 the core centriole structure is slightly shorter (Franz et al., 2013).

The CP110/Cep97 complex influences the Plk4 oscillation at the proximal end of the growing daughter centriole

To test whether the CP110/Cep97 complex influences cartwheel growth by altering the centriolar Plk4 oscillation, we examined the Plk4-GFP oscillations in nuclear cycle 12 in embryos where CP110 or Cep97 were either absent or were individually overexpressed. These perturbations altered both the amplitude and phase of the Plk4 oscillation at the proximal end of the growing daughter (Fig. 7).

We previously showed that in WT embryos the Plk4 oscillation exhibits adaptive behaviour: as its amplitude tends to decrease from nuclear cycle 11 to 13 , so its period tends to increase-and we hypothesised that the progressively decreasing amplitude leads to the progressively decreasing growth rate, while the progressively increasing period leads to the progressively increasing cartwheel growth period (Aydogan et al., 2020). Strikingly, the lack of CP110 or Cep97 led to an increase in the amplitude of the Plk4 oscillation-consistent with the faster rate of cartwheel growth we observe (Fig. 6)—but the peak of the Plk4 oscillation was shifted to later in Sphase, even though the cartwheels in these embryos grow for a shorter 
period. Similarly, while the overexpression of CP110 or Cep97 led to a decrease in the amplitude of the Plk4 oscillation-consistent with the slower rate of cartwheel growth we observe (Fig. 6)—-the peak of the Plk4 oscillation was shifted to earlier in S-phase, even though the cartwheels in these embryos grow for a longer period (see Discussion).

Altering the levels of the CP110/Cep97 complex does not detectably alter the cytoplasmic levels of Plk4, or vice versa, in embryos

CP110/Cep97 and Plk4 are normally localised to opposite ends of the growing centriole, so how can altering the levels of CP110/Cep97 influence centriolar Plk4? We wanted to test whether CP110/Cep97 might influence Plk4 abundance in the cytoplasm. As described previously, in our hands the cytosolic concentration of Plk4-NG expressed from its own promoter was too low to be measured by conventional Fluorescence Correlation Spectroscopy (FCS), so we used Peak Counting Spectroscopy (PeCoS) instead (Aydogan et al., 2020). Varying the cytoplasmic dosage of CP110 or Cep97 did not detectably alter cytoplasmic Plk4 levels (Fig. 8A). In addition, we used FCS and western blotting to measure the cytoplasmic concentration or total amount, respectively, of uCP110-GFP and uGFP-Cep97 in Plk4 $4^{1 / 2}$ embryos or in embryos carrying a previously described mutated form of Plk4 with reduced kinase activity (Plk4 ${ }^{R K A}$ ) (Aydogan et al., 2018). Neither the levels nor the kinase activity of Plk4 appeared to influence CP110 and Cep97 levels (Fig. 8, B and C). Thus, any crosstalk between CP110/Cep97 and Plk4 does not appear to rely on their ability to influence each other's cytoplasmic abundance in embryos. 


\section{Discussion}

Here we show that CP110 and Cep97 are recruited in an oscillatory manner to, or very close to, the distal end of the growing daughter centriole MTs. The CP110/Cep97 oscillation, however, does not appear to play a major part in determining the period of daughter centriole growth, and our findings essentially rule out the possibility that centriole MTs stop growing when a threshold level of CP110/Cep97 accumulates at the centriole distal end. Thus, although in many systems the centriole MTs are dramatically elongated in the absence of CP110 or Cep97 (Dobbelaere et al., 2020; Franz et al., 2013; Kohlmaier et al., 2009; Schmidt et al., 2009; Sharma et al., 2021; Winey and O'Toole, 2014), we suspect that this is largely due to a failure to properly "cap" these MTs after they have finished growing, rather than because the centriole MTs grow too quickly as the new daughter centriole is being assembled.

CP110 and Cep97 do not oscillate at centrioles because the proteins reach saturating levels on the centriole MTs and then unbind, as the amount of CP110/Cep97 recruited to centrioles is dramatically increased when either protein is overexpressed. It is unclear how these proteins interact specifically with the distal-ends of the centriole MTs, but we conclude that their binding sites are normally far from saturated, at least in the rapidly cycling Drosophila embryo. Importantly, the period of the CP110/Cep97 oscillation is strongly influenced by the activity of the core Cdk/Cyclin cell cycle oscillator (CCO). We suspect, therefore, that the CP110/Cep97 oscillation in these embryos may simply reflect the ability of these proteins to bind to centrioles when 
Cdk/Cyclin activity is low, but not when it is high. CP110 was originally identified as a CDK substrate (Chen et al., 2002), and presumably the CCO modifies (e.g., by phosphorylating) the centriolar CP110/Cep97 recruiting factor(s) and/or CP110/Cep97 to inhibit their recruitment. It is presently unclear why such a cell-cycle dependent regulation may be important to ensure that CP110/Cep97 cannot bind to the centriole distal ends during mitosis.

Perhaps surprisingly, we show that CP110/Cep97 levels can influence the growth of the centriole cartwheel, at least in part, by altering the parameters of the Plk4 oscillation at the base of the growing daughter centrioles. This reveals an unexpected crosstalk between proteins that are usually thought to influence events at the proximal end of the cartwheel and at the distal end of the centriole MTs. Although we currently do not understand how CP110/Cep97 levels influence Plk4 behaviour at the proximal end, our data suggests these proteins do not alter each other's cytoplasmic abundance. While CP110 and Cep97 are not essential for normal centriole duplication in mice and flies (Dobbelaere et al., 2020; Franz et al., 2013; Yadav et al., 2016), CP110 is required for Plk4-induced centriole overduplication in cultured human cells (Kleylein-Sohn et al., 2007), where Plk4 can also interact with and phosphorylate CP110 to promote centriole duplication (Lee et al., 2017). Thus, although the physiological significance of this crosstalk is not entirely clear, the crosstalk may be conserved in other species. 
It is important to note that changing the levels of CP110/Cep97 influences the

Plk4 oscillation in a surprising way. In the absence of CP110/Cep97, the cartwheel grows faster and for shorter period, but the Plk4 oscillation has a higher amplitude, and a longer period. Our previous observations would suggest that faster centriole growth for a shorter period would be associated with Plk4 oscillation that has a higher amplitude, but a shorter period (Aydogan et al., 2020). One way to explain this conundrum is if Plk4 is more active in the absence of CP110/Cep97-so the cartwheel would be built faster, but for a shorter period (Aydogan et al., 2020), as we observe-but the inactivated Plk4 is not efficiently released from its centriolar receptors (so Plk4 would accumulate at centrioles to a higher level and for a longer period). Clearly further work is required to understand how the Plk4 oscillation drives cartwheel assembly, and how this process is influenced by CP110/Cep97.

\section{Acknowledgements:}

We thank the members of Aydogan and Raff laboratories for critically reading the manuscript. Super-resolution microscopy was carried out at the Micron Oxford Advanced Bioimaging Unit, funded by a Strategic Award from the Wellcome Trust (107457). The research was funded by a Wellcome Trust Senior Investigator Award (104575 and 215523; T.L.S., M. M., S. S., A. W. and J.W.R.), an Edward Penley Abraham Scholarship (M.G.A.), a Sandler Foundation Investigator Award (M.G.A.), a UCSF PBBR New Frontiers Research Award (M.G.A.), a Wellcome Trust PhD Studentship (203855; L.E.H.), and a Ludwig Institute for Cancer Research funding (F.Y.Z.). 


\section{Author contributions:}

This study was conceptualised by M.G.A., L.E.H. and J.W.R. Investigation was done by M.G.A., L.E.H., T.L.S., M.M., S.S. and A.W. Data were analysed by M.G.A., L.E.H., T.L.S. and A.W. Methodology was developed by M.G.A., L.E.H. and J.W.R. Project was administrated by M.G.A., L.E.H. and J.W.R. Resources were shared/made by M.G.A., L.E.H., T.L.S., M.M., S.S., S.S.W., X.L. and F.Y.Z. Software work was carried out by M.G.A., L.E.H. and F.Y.Z. Overall supervision was done by M.G.A. and J.W.R. Validation experiments/analyses were carried out by M.G.A., L.E.H., T.L.S and S.S. Finally, M.G.A., L.E.H. and J.W.R. wrote, reviewed and edited the manuscript with input from all authors.

\section{Competing interests:}

Authors declare no competing interests for this study.

\section{Data, material and code availability:}

The script to automatize the CP110- and Cep97-GFP ring measurements analysis (of the super-resolution microscopy data) is available in the following link: <https://github.com/RaffLab/SIM-centriole-ring-measurement>. Rest of the data is available upon request. 


\section{References}

Alvarez-Rodrigo, I., Steinacker, T.L., Saurya, S., Conduit, P.T., Baumbach, J., Novak, Z.A., Aydogan, M.G., Wainman, A., and Raff, J.W. (2019). Evidence that a positive feedback loop drives centrosome maturation in fly embryos. ELife 8, e50130.

Arquint, C., and Nigg, E.A. (2016). The PLK4-STIL-SAS-6 module at the core of centriole duplication. Biochemical Society Transactions 44, 1253-1263.

Aydogan, M.G., Wainman, A., Saurya, S., Steinacker, T.L., Caballe, A., Novak, Z.A., Baumbach, J., Muschalik, N., and Raff, J.W. (2018). A homeostatic clock sets daughter centriole size in flies. J Cell Biol 217, 12331248.

Aydogan, M.G., Steinacker, T.L., Mofatteh, M., Wilmott, Z.M., Zhou, F.Y., Gartenmann, L., Wainman, A., Saurya, S., Novak, Z.A., Wong, S.-S., et al. (2020). An Autonomous Oscillation Times and Executes Centriole Biogenesis. Cell 181, 1566-1581.e27.

Banterle, N., and Gönczy, P. (2017). Centriole Biogenesis: From Identifying the Characters to Understanding the Plot. Annual Review of Cell and Developmental Biology 33, 23-49.

Basto, R., Brunk, K., Vinadogrova, T., Peel, N., Franz, A., Khodjakov, A., and Raff, J.W. (2008). Centrosome Amplification Can Initiate Tumorigenesis in Flies. Cell 133, 1032-1042.

Baumbach, J., Novak, Z.A., Raff, J.W., and Wainman, A. (2015). Dissecting the Function and Assembly of Acentriolar Microtubule Organizing Centers in Drosophila Cells In Vivo. PLoS Genet 11.

Breslow, D.K., and Holland, A.J. (2019). Mechanism and Regulation of Centriole and Cilium Biogenesis. Annual Review of Biochemistry 88, 691724.

Chang, C.-W., Hsu, W.-B., Tsai, J.-J., Tang, C.-J.C., and Tang, T.K. (2016). CEP295 interacts with microtubules and is required for centriole elongation. $J$ Cell Sci 129, 2501-2513.

Chen, Z., Indjeian, V.B., McManus, M., Wang, L., and Dynlacht, B.D. (2002). CP110, a Cell Cycle-Dependent CDK Substrate, Regulates Centrosome Duplication in Human Cells. Developmental Cell 3, 339-350.

Conduit, P.T., Wainman, A., Novak, Z.A., Weil, T.T., and Raff, J.W. (2015). Re-examining the role of Drosophila Sas-4 in centrosome assembly using two-colour-3D-SIM FRAP. ELife 4, e08483.

Dahl, K.D., Sankaran, D.G., Bayless, B.A., Pinter, M.E., Galati, D.F., Heasley, L.R., Giddings, T.H., and Pearson, C.G. (2015). A Short CEP135 Splice Isoform Controls Centriole Duplication. Current Biology 25, 2591-2596. 
Delgehyr, N., Rangone, H., Fu, J., Mao, G., Tom, B., Riparbelli, M.G., Callaini, G., and Glover, D.M. (2012). KIp10A, a Microtubule-Depolymerizing Kinesin13, Cooperates with CP110 to Control Drosophila Centriole Length. Current Biology 22, 502-509.

Dobbelaere, J., Josué, F., Suijkerbuijk, S., Baum, B., Tapon, N., and Raff, J. (2008). A Genome-Wide RNAi Screen to Dissect Centriole Duplication and Centrosome Maturation in Drosophila. PLOS Biology 6, e224.

Dobbelaere, J., Schmidt Cernohorska, M., Huranova, M., Slade, D., and Dammermann, A. (2020). Cep97 Is Required for Centriole Structural Integrity and Cilia Formation in Drosophila. Current Biology 30, 3045-3056.e7.

Farrell, J.A., and O'Farrell, P.H. (2014). From Egg to Gastrula: How the Cell Cycle Is Remodeled During the Drosophila Mid-Blastula Transition. Annual Review of Genetics 48, 269-294.

Firat-Karalar E. N. and Stearns T. (2014). The centriole duplication cycle. Philosophical Transactions of the Royal Society B: Biological Sciences 369, 20130460.

Franz, A., Roque, H., Saurya, S., Dobbelaere, J., and Raff, J.W. (2013). CP110 exhibits novel regulatory activities during centriole assembly in Drosophila. J Cell Biol 203, 785-799.

Gartenmann, L., Wainman, A., Qurashi, M., Kaufmann, R., Schubert, S., Raff, J.W., and Dobbie, I.M. (2017). A combined 3D-SIM/SMLM approach allows centriole proteins to be localized with a precision of $\sim 4-5 \mathrm{~nm}$. Current Biology 27, R1054-R1055.

Gemble, S., and Basto, R. (2018). Fast and furious . . or not, Plk4 dictates the pace. J Cell Biol jcb.201802084.

Guennec, M.L., Klena, N., Gambarotto, D., Laporte, M.H., Tassin, A.-M., Hoek, H. van den, Erdmann, P.S., Schaffer, M., Kovacik, L., Borgers, S., et al. (2020). A helical inner scaffold provides a structural basis for centriole cohesion. Science Advances 6, eaaz4137.

Guichard, P., Hachet, V., Majubu, N., Neves, A., Demurtas, D., Olieric, N., Fluckiger, I., Yamada, A., Kihara, K., Nishida, Y., et al. (2013). Native architecture of the centriole proximal region reveals features underlying its 9fold radial symmetry. Curr. Biol. 23, 1620-1628.

Kleylein-Sohn, J., Westendorf, J., Le Clech, M., Habedanck, R., Stierhof, Y.D., and Nigg, E.A. (2007). Plk4-Induced Centriole Biogenesis in Human Cells. Developmental Cell 13, 190-202.

Kohlmaier, G., Lončarek, J., Meng, X., McEwen, B.F., Mogensen, M.M., Spektor, A., Dynlacht, B.D., Khodjakov, A., and Gönczy, P. (2009). Overly Long Centrioles and Defective Cell Division upon Excess of the SAS-4Related Protein CPAP. Current Biology 19, 1012-1018. 
Kong, D., Sahabandu, N., Sullenberger, C., Vásquez-Limeta, A., Luvsanjav, D., Lukasik, K., and Loncarek, J. (2020). Prolonged mitosis results in structurally aberrant and over-elongated centrioles. Journal of Cell Biology 219.

Lee, M., Seo, M.Y., Chang, J., Hwang, D.S., and Rhee, K. (2017). PLK4 phosphorylation of CP110 is required for efficient centriole assembly. Cell Cycle 16, 1225-1234.

Lin, Y.-C., Chang, C.-W., Hsu, W.-B., Tang, C.-J.C., Lin, Y.-N., Chou, E.-J., Wu, C.-T., and Tang, T.K. (2013). Human microcephaly protein CEP135 binds to hSAS- 6 and CPAP, and is required for centriole assembly. The EMBO Journal 32, 1141-1154.

Liu, B., Zhao, H., Wu, K., and Großhans, J. (2021). Temporal Gradients Controlling Embryonic Cell Cycle. Biology 10, 513.

Liu, T.-L., Upadhyayula, S., Milkie, D.E., Singh, V., Wang, K., Swinburne, I.A., Mosaliganti, K.R., Collins, Z.M., Hiscock, T.W., Shea, J., et al. (2018). Observing the cell in its native state: Imaging subcellular dynamics in multicellular organisms. Science 360, eaaq1392.

Mukherji, S., and O'Shea, E.K. (2014). Mechanisms of organelle biogenesis govern stochastic fluctuations in organelle abundance. ELife 3, e02678.

Novak, Z.A., Conduit, P.T., Wainman, A., and Raff, J.W. (2014). Asterless Licenses Daughter Centrioles to Duplicate for the First Time in Drosophila Embryos. Current Biology 24, 1276.

Saurya, S., Roque, H., Novak, Z.A., Wainman, A., Aydogan, M.G., Volanakis, A., Sieber, B., Pinto, D.M.S., and Raff, J.W. (2016). Drosophila Ana1 is required for centrosome assembly and centriole elongation. J Cell Sci 129, 2514-2525.

Schmidt, T.I., Kleylein-Sohn, J., Westendorf, J., Le Clech, M., Lavoie, S.B., Stierhof, Y.-D., and Nigg, E.A. (2009). Control of Centriole Length by CPAP and CP110. Current Biology 19, 1005-1011.

Sharma, A., Aher, A., Dynes, N.J., Frey, D., Katrukha, E.A., Jaussi, R., Grigoriev, I., Croisier, M., Kammerer, R.A., Akhmanova, A., et al. (2016). Centriolar CPAP/SAS-4 Imparts Slow Processive Microtubule Growth. Developmental Cell 37, 362-376.

Sharma, A., Olieric, N., and Steinmetz, M.O. (2021). Centriole length control. Current Opinion in Structural Biology 66, 89-95.

Spektor, A., Tsang, W.Y., Khoo, D., and Dynlacht, B.D. (2007). Cep97 and CP110 Suppress a Cilia Assembly Program. Cell 130, 678-690.

Tinevez, J.-Y., Perry, N., Schindelin, J., Hoopes, G.M., Reynolds, G.D., Laplantine, E., Bednarek, S.Y., Shorte, S.L., and Eliceiri, K.W. (2017). 
TrackMate: An open and extensible platform for single-particle tracking.

Methods 115, 80-90.

Waithe, D., Clausen, M.P., Sezgin, E., and Eggeling, C. (2016). FoCuS-point: software for STED fluorescence correlation and time-gated single photon counting. Bioinformatics 32, 958-960.

Winey, M., and O'Toole, E. (2014). Centriole structure. Philosophical Transactions of the Royal Society B: Biological Sciences 369, 20130457.

Yadav, S.P., Sharma, N.K., Liu, C., Dong, L., Li, T., and Swaroop, A. (2016). Centrosomal protein CP110 controls maturation of the mother centriole during cilia biogenesis. Development 143, 1491-1501.

Yang, T.T., Chong, W.M., Wang, W.-J., Mazo, G., Tanos, B., Chen, Z., Tran, T.M.N., Chen, Y.-D., Weng, R.R., Huang, C.-E., et al. (2018). Superresolution architecture of mammalian centriole distal appendages reveals distinct blade and matrix functional components. Nature Communications 9 , 2023.

Zheng, X., Ramani, A., Soni, K., Gottardo, M., Zheng, S., Ming Gooi, L., Li, W., Feng, S., Mariappan, A., Wason, A., et al. (2016). Molecular basis for CPAP-tubulin interaction in controlling centriolar and ciliary length. Nature Communications 7, 11874. 


\section{Materials and Methods}

\section{Drosophila melanogaster stocks and husbandry}

D. melanogaster stocks used in this study are listed in Supplementary Table

1 , and the lines generated and tested here are listed in Supplementary Table

2. To generate the Ubq-CP110 (uCP110) construct, a stop codon was introduced into a previously generated pDONR-CP110L (containing a full length CP110 cDNA) (Franz et al., 2013) by site directed mutagenesis using Quikchange II XL mutagenesis kit (Agilent technologies). This was then recombined with the pUbq-empty vector (This paper; details available on request). To generate the Ubq-Cep97 (uCep97) construct, the pZeo-CG3980NT vector (Dobbelaere et al., 2008) was directly recombined with the pUbqempty vector.

The pDONR-Zeo-eCP110 cDNA construct was cloned by assembling two fragments: the $\sim 2 \mathrm{~kb}$ region upstream of the cp110 start codon and the pDONR-Zeo-CP110 cDNA vector containing the long isoform of CP110 minus the stop codon (Franz et al., 2013), using NEBuilder HiFi assembly (NEB). The cDNA construct pDONR-Zeo-eCP110 was then recombined with an mGFP-CT empty destination vector (pNoP-mGFP-CT-DEST; This paper details available upon request) via Gateway Technology (Thermo Fisher Scientific). The pNoP-mGFP-CT-DEST was made by removing the Ubiquitin promoter from a previously published (Basto et al., 2008) Ubq-mGFP-CT destination vector.

Primer sequences used to introduce a stop codon for the uCP110 construct, to amplify the $c p 110$ promoter region, to amplify the pDONR-Zeo vector containing the cp110 sequence, and to clone the C-terminal fragment aa 329807 of Cep97 into the pDONR vector are listed in Supplementary Table 3. Transgenic flies were generated by the Fly Facility in the Department of Genetics, University of Cambridge (UK).

Flies were maintained at $18^{\circ} \mathrm{C}$ or $25^{\circ} \mathrm{C}$ on Drosophila culture medium $(0.77 \%$ agar, $6.9 \%$ maize, $0.8 \%$ soya, $1.4 \%$ yeast, $6.9 \%$ malt, $1.9 \%$ molasses, $0.5 \%$ propionic acid, $0.03 \%$ ortho-phosphoric acid and $0.3 \%$ nipagin) in vials or in bottles. For embryo collections, $25 \%$ cranberry-raspberry juice plates $(2 \%$ sucrose and $1.8 \%$ agar with a drop of yeast suspension) were used.

\section{Embryo collections}

For all imaging experiments, embryos were collected for $1 \mathrm{~h}$ at $25^{\circ} \mathrm{C}$, and then aged for $45 \mathrm{~min}$ to $1 \mathrm{~h}$. Before imaging, embryos were dechorionated by hand, mounted on a strip of glue painted on a $35 \mathrm{~mm}$ glass bottom petri dish with 14 $\mathrm{mm}$ micro-well (MatTek) and desiccated for $1 \mathrm{~min}$ at $25^{\circ} \mathrm{C}$. Embryos were then covered with Voltalef ${ }^{\circledR}$ oil (ARKEMA).

\section{Hatching experiments}


In order to measure embryo hatching rates, 0-3 h embryos were collected and aged for $24 \mathrm{~h}$, and the \% of embryos that hatched out of their chorion was scored. 5 technical repeats were carried out over multiple days, and at least 120 embryos were analysed for each genotype per repeat.

\section{Image acquisition, processing and analysis}

\section{Airy-scan super resolution microscopy}

Living embryos were imaged using an inverted Zeiss 880 microscope fitted with an airy-scan detector. The system was equipped with Plan-Apochromat $63 x / 1.4$ NA oil lens. $488 \mathrm{~nm}$ argon and $561 \mathrm{~nm}$ diode lasers were used to excite GFP and RFP (or mCherry) respectively. Stacks of 5 slices at $0.2 \mu \mathrm{m}$ intervals were collected with a zoom value of 24.41 pixels $/ \mu \mathrm{m}$. Focus was readjusted in between image collection. Images were airy-processed in 3D with a strength value of Auto ( 6) or 6.5.

Fluorescence Recovery After Photobleaching (FRAP) experiments were performed on the same Zeiss 880 system. Settings for the sequence of events needed for FRAP experiments were as follows: (1) Acquisition of a single Z-stack in Airy-scan mode (Pre-bleach in Fig. S2A); (2) multi-spot serial photo-bleaching (4 regions at a time); (3) acquisition of the photo-bleached image in Airy-scan mode (Bleach in Fig. S2A); (4) acquisition of the postbleach images in Airy-scan mode (Post-bleach in Fig. S2A).

This protocol was also used to examine the site where Sas-6-GFP incorporates into centrioles in WT, CP110-/- and Cep97-/- embryos (Fig. S2B). For this analysis, embryos exiting mitosis of nuclear Cycle 13 were identified and daughter centrioles were allowed to grow for 6 minutes into Cycle 14allowing centrioles to grow to approximately half of their final size (Aydogan et al., 2018). The site of new Sas-6-GFP recruitment was then determined using a previously described pipeline (Aydogan et al., 2018). This analysis was performed blind.

\section{Spinning Disk Confocal Microscopy}

Living embryos were imaged using either a Perkin Elmer ERS Spinning Disk confocal system on a Zeiss Axiovert 200M microscope equipped with PlanApochromat $63 x / 1.4$ NA oil DIC lens, or an EM-CCD Andor iXon+ camera on a Nikon Eclipse TE200-E microscope with a Plan-Apochromat 60x/1.42 NA oil DIC lens. 488 and $561 \mathrm{~nm}$ lasers were used to excite GFP and RFP (or mCherry) respectively. Confocal sections of 13 (Perkin Elmer) or 17 (Andor) slices with $0.5 \mu \mathrm{m}$ thick intervals were collected every $30 \mathrm{sec}$.

Post-acquisition image processing (including image projection, photobleaching correction and background subtraction) was carried out using Fiji (NIH, US) as described previously (Aydogan et al., 2018). uCP110-GFP, eCP110-GFP, uGFP-Cep97, eCep97-GFP, PIk4-GFP, uRFP-Cep97 and Sas6-GFP foci were tracked using TrackMate (Tinevez et al., 2017), a plug-in of Fiji, with track spot diameter size of $1.1 \mu \mathrm{m}$. The regressions for Plk4-GFP 
oscillations and Sas-6-GFP cartwheel growth curves were calculated in Prism 8 (GraphPad Software), as described previously (Aydogan et al., 2018). The regressions for UGFP-Cep97 and eCep97-GFP (or, uCP110-GFP and eCP110-GFP) were calculated using the nonlinear regression (curve fit) function in Prism 8. Discrete curves in S-phase were first fitted against four different functions to decide the best regression model: 1) Lorentzian, 2) Gaussian, 3) Increase - Constant - Decrease, and 4) Increase - Decrease. Among these models, Lorentzian best fit the data (data not shown). Thus, all the fluorescently tagged Cep97 and CP110 curves in S-phase were regressed with the Lorentzian function. The Lorentzian and Gaussian functions are integral to Prism 8, while the latter two functions were described previously (Alvarez-Rodrigo et al., 2019). In order to plot the dynamics of uCP110-GFP and URFP-Cep97 together, the highest mean fluorescence signal for each tag in nuclear cycle 12 was scaled to 1 A.U., and this scaling factor was accordingly applied across all cycles.

As previously described (Aydogan et al., 2018), the beginning of S-phase was taken as the time of centrosome separation ("CS"). Entry into mitosis was taken as the time of nuclear envelope breakdown (NEB).

\section{$\underline{\text { 3D-Structured Illumination Microscopy (3D-SIM) }}$}

Living embryos were imaged at $21^{\circ} \mathrm{C}$ using a DeltaVision OMX V3 Blaze microscope (GE Healthcare Life Sciences, UK). The system was equipped with a 60x/1.42 NA oil UPlanSApo objective (Olympus Corp.), $488 \mathrm{~nm}$ and $593 \mathrm{~nm}$ diode lasers and Edge $5.5 \mathrm{sCMOS}$ cameras (PCO). All image acquisition and post-processing was performed as described previously (Aydogan et al., 2018, 2020). In order to investigate where CP110 and Cep97 radially localizes on the centriole, wing disc preparations were made as previously described (Gartenmann et al., 2017) and imaged with an OMX V2 microscope (GE Healthcare Life Sciences, UK) with a 100x/1.4 NA oil objective (UPlanSApo, Olympus), 488nm diode (Sapphire 488-200, Coherent) and 592nm (F-04306-01, MPB Communications) fiber lasers and Evolve 512 Delta C EMCCD cameras (Photometrics). The orientation of the centrioles and the radial localisation of the various CP110 and Cep97 constructs was assessed following a previously described protocol (Gartenmann et al., 2017) and using our own python code (available at: https://github.com/RaffLab/SIMcentriole-ring-measurement). Briefly, the rings of both Asl-mCherry and various GFP tagged CP110/Cep97 markers were fitted with an elliptical annular Gaussian profile, obtaining fit parameters for the major and minor axis. Centrioles that had Asl and CP110/Cep97 ring eccentricity (major:minor axis ratio) of less than 1.2 were considered well oriented. The width of the resulting Gaussian fit for CP110/Cep97 was then measured.

\section{Immunoblotting}

Immunoblotting was carried out as described previously (Aydogan et al., 2018). For all blots, 15 staged early embryos were boiled in sample buffer and loaded in each lane. Primary antibodies used in this study are as follows: rabbit anti-CP110 (Franz et al., 2013), rabbit anti-Cep97 (Dobbelaere et al., 
2020), rabbit anti-Cnn (Dobbelaere et al., 2008) and mouse anti-Actin

(Sigma); all primary antibodies were used at 1:500 dilution in blocking solution (Aydogan et al., 2018). Primary antibody incubation period was $1 \mathrm{~h}$. Secondary antibodies used in this study are as follows: HRPO-linked anti-mouse or antirabbit IgG (both GE Healthcare); both the secondary antibodies were used at 1:3000 dilution in blocking solution (Aydogan et al., 2018).

The quantification of Western blots was carried out in ImageJ. Briefly, the endogenous Cep97 or CP110 signal in all conditions was selected by using the Rectangle tool to create a region of interest (ROI). The detected signals in each lane were plotted using Plot Lanes option from the Gels tab. The area under the curve for each lane was then calculated and exported to GraphPad Prism 8. The same size ROI was used to repeat the process for the loading control (the endogenous Actin signal) in each lane. The CP110 or Cep97 signals were normalized to the signal of the loading control in each lane, then to the respective mean value of CP110 or Cep97 signals overall.

\section{Fluorescence Correlation Spectroscopy (FCS)}

FCS measurements were obtained as previously described (Aydogan et al., 2020). Every measurement consisted of $6 \times 10$ sec point recordings, which were acquired around the centriolar plane (near the embryo cortex) at the beginning of nuclear cycle 12 . The laser power was kept constant at $6.31 \mu \mathrm{W}$. All recordings were fitted with 8 previously described diffusion models (Aydogan et al., 2020) within the boundaries of $4 \times 10^{-4}-2.1 \times 10^{3} \mathrm{~ms}$ using FoCuS-point (Waithe et al., 2016). The diffusion parameters were restricted to a minimal mean residence time of $0.7 \mathrm{~ms}$, and the anomalous subdiffusion $\alpha$ and the spatial description of the excitation volume $A R$ were kept constant at 0.7 and 5, respectively. The preferred model was chosen based on the Bayesian Information Criterion (BIC), which in our case was one diffusing species with one blinking and one triplet state of the fluorophore (Model 4). Afterwards, the cytoplasmic concentrations were corrected for the background noise (through point FCS measurements in $n=21$ WT embryos), and a ROUT outlier test $(\mathrm{Q}=1 \%)$ was performed on all 10 -sec long concentration measurements. The outlier tests were performed before any further statistical tests were applied. Only the measurements with four or more recordings were kept for statistical analysis.

\section{Peak Counting Spectroscopy (PeCoS)}

PeCoS measurements were obtained as previously described (Aydogan et al., 2020). $180 \mathrm{sec}$ long recordings were made at the same position and the same nuclear cycle stage as the FCS measurements. In addition to the measurements of embryos expressing Plk4-GFP under its own endogenous promoter, 12 control measurements were obtained from embryos expressing Asl-mKate2, which were used to determine the background auto-

fluorescence. The subtraction of their background (="Mean $+7{ }^{*} S D$ ") resulted in an average peak count of 4.5 (which fulfilled the requirement of less than 5 peaks per 180 sec-long control recording), and it was therefore chosen as background threshold for all in vivo measurements. The ROUT outlier test $(Q$ 
$=1 \%$ ) was performed before further statistical tests were applied.

\section{Quantification and statistical analysis}

All the details for quantification, statistical tests, $\mathrm{n}$ numbers, definitions of center, and dispersion and precision measures were either described in the main text, relevant figure legends or in Materials and Methods section. Significance in statistical tests was defined by $p<0.05$. To determine whether the data values came from a Gaussian distribution, D'Agostino-Pearson omnibus normality test was applied. GraphPad Prism 7 or 8 were used for all the modeling and statistical analyses, unless otherwise stated. 


\section{Supplementary Tables}

Table S1: D. melanogaster alleles used in this study.

\begin{tabular}{|c|c|c|}
\hline Allele* & $\begin{array}{l}\text { Source } \\
\text { (reference \#) }\end{array}$ & ID \\
\hline Ubq-GFP-Cep97 & $\begin{array}{l}\text { (Dobbelaere et } \\
\text { al., 2008) }\end{array}$ & FlyBase ID: FBal0343980 \\
\hline Ubq-CP110-GFP & $\begin{array}{l}\text { (Dobbelaere et } \\
\text { al., 2008) }\end{array}$ & FlyBase ID: FBtp0092320 \\
\hline Ubq-CP110 & This paper & $\mathrm{N} / \mathrm{A}$ \\
\hline Ubq-Cep97 & This paper & $\mathrm{N} / \mathrm{A}$ \\
\hline Cep97-GFP & $\begin{array}{l}\text { (Dobbelaere et } \\
\text { al., 2020) }\end{array}$ & FlyBase ID: FBal0362412 \\
\hline CP110-GFP & This paper & $\mathrm{N} / \mathrm{A}$ \\
\hline Plk4-GFP & $\begin{array}{l}\text { (Aydogan et al., } \\
\text { 2018) }\end{array}$ & FlyBase ID: FBal0343977 \\
\hline Ubq-RFP-Cep97 & $\begin{array}{l}\text { (Dobbelaere et } \\
\text { al., 2008) }\end{array}$ & N/A \\
\hline Plk4 ${ }^{\mathrm{Aa} 74}$ & $\begin{array}{l}\text { (Aydogan et al., } \\
\text { 2018) }\end{array}$ & FlyBase ID: FBab0049012 \\
\hline CP110 $\triangle$ & $\begin{array}{l}\text { (Franz et al., } \\
\text { 2013) }\end{array}$ & FlyBase ID: FBal0294119 \\
\hline Cep97 & 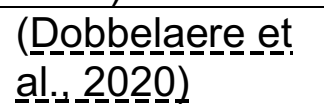 & FlyBase ID: FBal0362411 \\
\hline Asl-mCherry & $\begin{array}{l}\text { (Conduit et al., } \\
\text { 2015) }\end{array}$ & FlyBase ID: FBal0343645 \\
\hline Ubq-Cep97-GFP & $\begin{array}{l}\text { (Dobbelaere et } \\
\text { al., 2008) }\end{array}$ & $\mathrm{N} / \mathrm{A}$ \\
\hline Sas-6-GFP & $\begin{array}{l}\text { (Aydogan et al., } \\
2018 \text { ) }\end{array}$ & FlyBase ID: FBtp0131375 \\
\hline Plk4 & $\begin{array}{l}\text { (Aydogan et al., } \\
\text { 2018) }\end{array}$ & FlyBase ID: FBal0343978 \\
\hline Plk4 RKA & $\begin{array}{l}\text { (Aydogan et al., } \\
\text { 2018) }\end{array}$ & FlyBase ID: FBtp0131379 \\
\hline Asl-mKate2 & $\begin{array}{l}\text { (Aydogan et al., } \\
2020 \text { ) }\end{array}$ & FlyBase ID: FBal0366991 \\
\hline asil $\left.\right|^{\mathrm{B} 46}$ & $\begin{array}{l}\text { (Baumbach et } \\
\text { al., 2015) }\end{array}$ & FlyBase ID: FBal0343439 \\
\hline
\end{tabular}

*The alleles listed here were expressed under their endogenous promoters unless otherwise specified. 
Table S2: D. melanogaster strains generated and/or used in this study.

\begin{tabular}{|c|c|c|}
\hline Strain Genotype & Tissue & Type of experiment \\
\hline Ubq-GFP-Cep97 / + & Embryo & $\begin{array}{l}\text { Confocal microscopy; Western } \\
\text { blot; Fluorescence correlation } \\
\text { spectroscopy }\end{array}$ \\
\hline Ubq-CP110-GFP / + & Embryo & $\begin{array}{l}\text { Confocal microscopy; Western } \\
\text { blot; Fluorescence correlation } \\
\text { spectroscopy }\end{array}$ \\
\hline Cep97-GFP / + & Embryo & $\begin{array}{l}\text { Confocal microscopy; Western } \\
\text { blot }\end{array}$ \\
\hline CP110-GFP / + & Embryo & $\begin{array}{l}\text { Confocal microscopy; Western } \\
\text { blot }\end{array}$ \\
\hline $\begin{array}{l}\text { Ubq-CP110-GFP / +; Plk4 }{ }^{\mathrm{Aa} 74} / \\
+\end{array}$ & Embryo & $\begin{array}{l}\text { Fluorescence correlation } \\
\text { spectroscopy }\end{array}$ \\
\hline $\begin{array}{l}\text { Ubq-GFP-Cep97/ +; +/+; } \\
\text { Plk4 }\end{array}$ & Embryo & $\begin{array}{l}\text { Fluorescence correlation } \\
\text { spectroscopy }\end{array}$ \\
\hline Plk4-GFP / + & Embryo & $\begin{array}{l}\text { Confocal microscopy; Peak } \\
\text { counting spectroscopy }\end{array}$ \\
\hline $\begin{array}{l}\text { CP110 } / \text { CP110 } \Delta ; \text { Plk4-GFP / } \\
+\end{array}$ & Embryo & $\begin{array}{l}\text { Confocal microscopy; Peak } \\
\text { counting spectroscopy }\end{array}$ \\
\hline Plk4-GFP / Ubq-CP110 & Embryo & $\begin{array}{l}\text { Confocal microscopy; Peak } \\
\text { counting spectroscopy }\end{array}$ \\
\hline Plk4-GFP, Cep97 $\Delta$ / Cep97 $\Delta$ & Embryo & $\begin{array}{l}\text { Confocal microscopy; Peak } \\
\text { counting spectroscopy }\end{array}$ \\
\hline Plk4-GFP / Ubq-Cep97 & Embryo & $\begin{array}{l}\text { Confocal microscopy; Peak } \\
\text { counting spectroscopy }\end{array}$ \\
\hline Asl-mCherry / Ubq-CP110-GFP & $\begin{array}{l}\text { Embryo; } \\
\text { wing } \\
\text { discs }\end{array}$ & $\begin{array}{l}\text { 3D-SIM; Airy-scan super } \\
\text { resolution microscopy }\end{array}$ \\
\hline $\begin{array}{l}\text { Ubq-GFP-Cep97 / +; Asl- } \\
\text { mCherry / + }\end{array}$ & $\begin{array}{l}\text { Embryo; } \\
\text { wing } \\
\text { discs }\end{array}$ & $\begin{array}{l}\text { 3D-SIM; Airy-scan super } \\
\text { resolution microscopy }\end{array}$ \\
\hline $\begin{array}{l}\text { Asl-mCherry / +; Ubq-Cep97- } \\
\text { GFP / + }\end{array}$ & $\begin{array}{l}\text { Wing } \\
\text { discs }\end{array}$ & 3D-SIM \\
\hline
\end{tabular}


Table S2 Cont'd: $D$. melanogaster strains generated and/or used in this study.

\begin{tabular}{|c|c|c|}
\hline Strain Genotype & Tissue & Type of experiment \\
\hline Oregon- $R$ (Wild-type strain) & Embryo & $\begin{array}{l}\text { Western Blot; Hatching assay; } \\
\text { Fluorescence correlation } \\
\text { spectroscopy }\end{array}$ \\
\hline $\mathrm{CP} 110 \Delta / \mathrm{CP} 110 \Delta$ & Embryo & Western blot; Hatching assay \\
\hline Ubq-CP110 / + & Embryo & Western blot; Hatching assay \\
\hline Cep97 $/$ Cep97 & Embryo & Western blot; Hatching assay \\
\hline Ubq-Cep97 / + & Embryo & Western blot; Hatching assay \\
\hline $\begin{array}{l}\text { Ubq-CP110-GFP / } \\
\text { Ubq-RFP-Cep97 }\end{array}$ & Embryo & Confocal microscopy \\
\hline Sas-6-GFP / + & Embryo & $\begin{array}{l}\text { Confocal microscopy; Airy- } \\
\text { scan super resolution } \\
\text { microscopy }\end{array}$ \\
\hline $\begin{array}{l}\text { CP110 } \Delta / \text { CP110 } \Delta \text {; Sas-6-GFP } \\
/+\end{array}$ & Embryo & $\begin{array}{l}\text { Confocal microscopy; Airy- } \\
\text { scan super resolution } \\
\text { microscopy }\end{array}$ \\
\hline Sas-6-GFP / Ubq-CP110 & Embryo & Confocal microscopy \\
\hline Sas-6-GFP, Cep97 $/$ Cep97 $\Delta$ & Embryo & $\begin{array}{l}\text { Confocal microscopy; Airy- } \\
\text { scan super resolution } \\
\text { microscopy }\end{array}$ \\
\hline Sas-6-GFP / Ubq-Cep97 & Embryo & Confocal microscopy \\
\hline Asl-mCherry / Sas-6-GFP & Embryo & $\begin{array}{l}\text { Airy-scan super resolution } \\
\text { microscopy }\end{array}$ \\
\hline $\begin{array}{l}\text { CP110 } / \text { / CP110 } 1 \text {; Asl- } \\
\text { mCherry / Sas-6-GFP }\end{array}$ & Embryo & $\begin{array}{l}\text { Airy-scan super resolution } \\
\text { microscopy }\end{array}$ \\
\hline $\begin{array}{l}\text { Asl-mCherry, Cep97 / Sas-6- } \\
\text { GFP, Cep97 }\end{array}$ & Embryo & $\begin{array}{l}\text { Airy-scan super resolution } \\
\text { microscopy }\end{array}$ \\
\hline Ubq-GFP-Cep97 / +; Plk4 /+ & Embryo & $\begin{array}{l}\text { Fluorescence correlation } \\
\text { spectroscopy }\end{array}$ \\
\hline
\end{tabular}


Table S2 Cont'd: $D$. melanogaster strains generated and/or used in this study.

\begin{tabular}{|c|c|c|}
\hline Strain Genotype & Tissue & Type of experiment \\
\hline $\begin{array}{l}\text { Ubq-GFP-Cep97 / +; + /+; } \\
\text { Plk4 }{ }^{\text {RKA } /+}\end{array}$ & Embryo & $\begin{array}{l}\text { Fluorescence correlation } \\
\text { spectroscopy }\end{array}$ \\
\hline Ubq-CP110-GFP / Plk4 & Embryo & $\begin{array}{l}\text { Fluorescence correlation } \\
\text { spectroscopy }\end{array}$ \\
\hline Ubq-CP110-GFP / +; Plk4 RKA / + & Embryo & $\begin{array}{l}\text { Fluorescence correlation } \\
\text { spectroscopy }\end{array}$ \\
\hline Plk4 ${ }^{\text {Aa74 } /+~}$ & Embryo & Western blot \\
\hline Plk4 / + & Embryo & Western Blot \\
\hline $\mathrm{Plk}^{\mathrm{RKA}} /+$ & Embryo & Western blot \\
\hline Asl-mKate2, as| ${ }^{\mathrm{B} 46} /+$ & Embryo & Peak counting spectroscopy \\
\hline
\end{tabular}


Table S3: Oligonucleotides used in this study.

\begin{tabular}{|l|l|l|}
\hline Oligonucleotide name & Sequence & Source \\
\hline $\begin{array}{l}\text { Primer to introduce a stop } \\
\text { codon into pDONR-CP110: } \\
\text { Forward }\end{array}$ & $\begin{array}{l}\text { CAAACATCGCCGATT } \\
\text { GGATTAGGACCCAGC } \\
\text { TTTCTTGTAC }\end{array}$ & $\begin{array}{l}\text { Invitrogen, Thermo } \\
\text { Fisher Scientific }\end{array}$ \\
\hline $\begin{array}{l}\text { Primer to introduce a stop } \\
\text { codon into pDONR-CP110: } \\
\text { Reverse }\end{array}$ & $\begin{array}{l}\text { GTACAAGAAAGCTGG } \\
\text { GTCCTAATCCAATCG } \\
\text { GCGATGTTTG }\end{array}$ & $\begin{array}{l}\text { Invitrogen, Thermo } \\
\text { Fisher Scientific }\end{array}$ \\
\hline $\begin{array}{l}\text { Primer to clone the C- } \\
\text { terminal fragment aa 329- } \\
807 \text { of Cep97 into the } \\
\text { pDONR vector: } \\
\text { Forward }\end{array}$ & $\begin{array}{l}\text { GGGGACAAGTTTGTA } \\
\text { CAAAAAACAGGCTT } \\
\text { GTTCTCCCGCTTGAG } \\
\text { TGGCCGCCAGG }\end{array}$ & $\begin{array}{l}\text { Invitrogen, Thermo } \\
\text { Fisher Scientific }\end{array}$ \\
\hline $\begin{array}{l}\text { Primer to clone the C- } \\
\text { terminal fragment aa } 329- \\
\text { 807 of Cep97 into the } \\
\text { pDONR vector: } \\
\text { Reverse }\end{array}$ & $\begin{array}{l}\text { GGGGACCACTTTGTA } \\
\text { CAAGAAAGCTGGGTG } \\
\text { TCATGGATCTTTATCA } \\
\text { AGATTTTC }\end{array}$ & $\begin{array}{l}\text { Invitrogen, Thermo } \\
\text { Fisher Scientific }\end{array}$ \\
\hline $\begin{array}{l}\text { Primer to amplify the cp110 } \\
\text { promoter region: } \\
\text { Forward }\end{array}$ & $\begin{array}{l}\text { TGTACAAAAAAGCAG } \\
\text { GCTTCGTTCCCTTTC } \\
\text { GCTGTCAAG }\end{array}$ & $\begin{array}{l}\text { Invitrogen, Thermo } \\
\text { Fisher Scientific }\end{array}$ \\
\hline $\begin{array}{l}\text { Primer to amplify the cp110 } \\
\text { promoter region: } \\
\text { Reverse }\end{array}$ & $\begin{array}{l}\text { ATTGCCCACGTCGCA } \\
\text { TCCATTGGTGTTTTGC } \\
\text { TACTGGG }\end{array}$ & $\begin{array}{l}\text { Invitrogen, Thermo } \\
\text { Fisher Scientific }\end{array}$ \\
\hline $\begin{array}{l}\text { Primer to amplify the } \\
\text { pDONR-Zeo vector } \\
\text { containing the } c p 110 \\
\text { sequence: } \\
\text { Forward }\end{array}$ & $\begin{array}{l}\text { ATGGATGCGACGTGG } \\
\text { GCA }\end{array}$ & $\begin{array}{l}\text { Invitrogen, Thermo } \\
\text { Fisher Scientific }\end{array}$ \\
\hline $\begin{array}{l}\text { Primer to amplify the } \\
\text { pDONR-Zeo vector } \\
\text { containing the } c p 110 \\
\text { sequence: } \\
\text { Reverse }\end{array}$ & $\begin{array}{l}\text { GAAGCCTGCTTTTTTG } \\
\text { TAC }\end{array}$ & $\begin{array}{l}\text { Invitrogen, Thermo } \\
\text { Fisher Scientific }\end{array}$ \\
\hline
\end{tabular}




\section{Figures and legends}

\section{Figure 1}

A
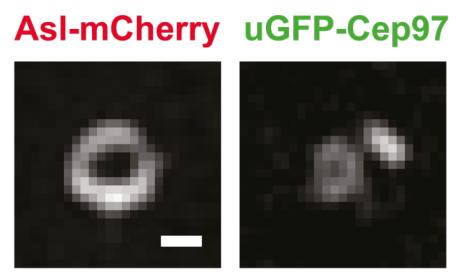

Asl-mCherry
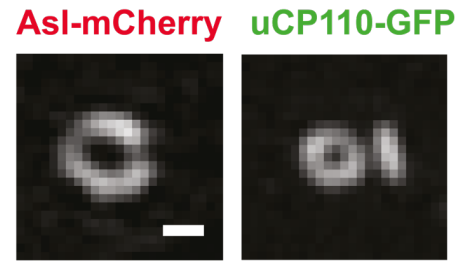

Merge

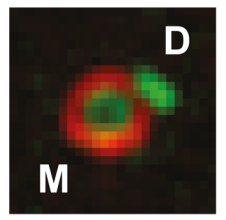

Merge

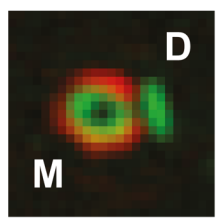

Mother

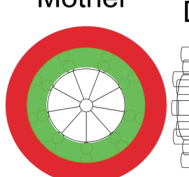

Daughter

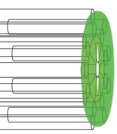

C

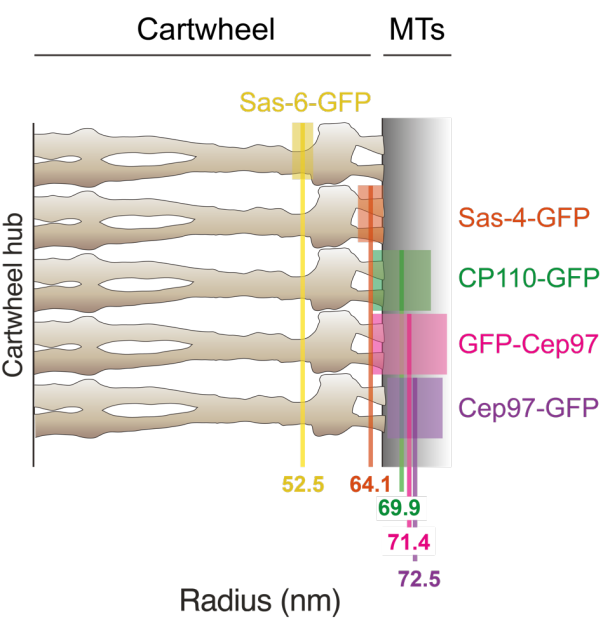

B

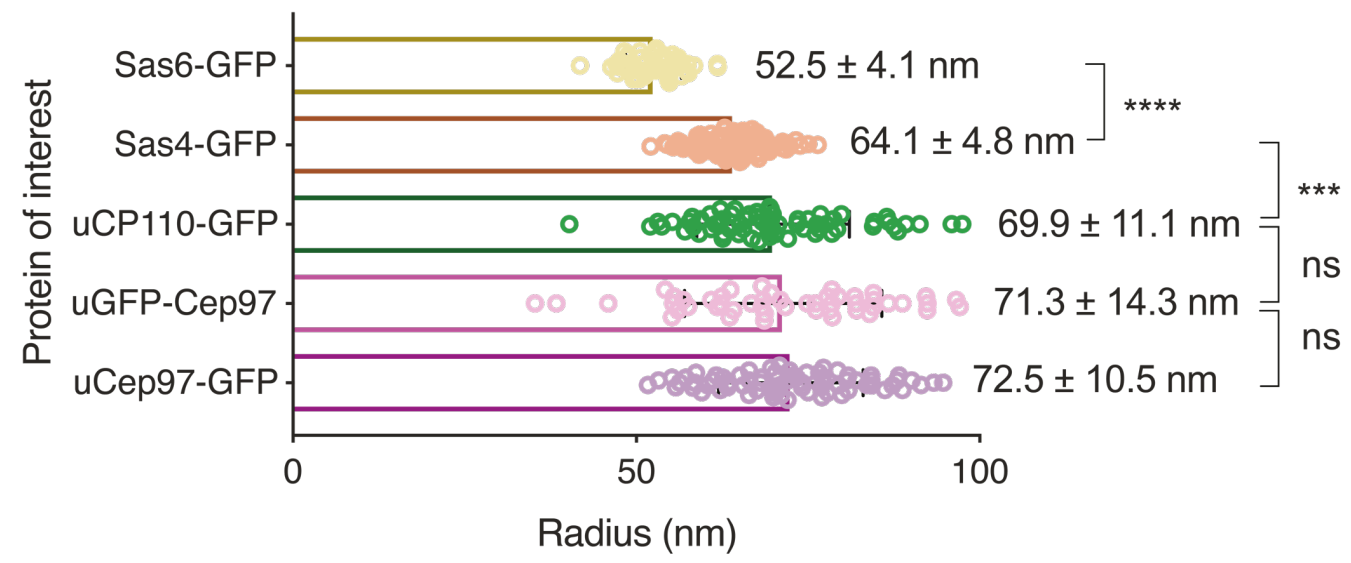

Figure 1. 3D-SIM analyses reveal that CP110 and Cep97 co-localise near the distal-end of the centriole microtubules.

(A) 3D-SIM micrographs show the distribution of the mother centriole marker Asl-mCherry and either uCep97-GFP or uCP110-GFP at mother/daughter centriole pairs in Drosophila wing disc cells (Scale bars $=0.2 \mu \mathrm{m}$ ). uCep97GFP and uCP110-GFP are located at the distal ends of the mother and daughter centrioles (with the mother centriole viewed "end-on", as depicted in the schematic). (B) The horizontal bar chart quantifies the mean radii of the indicated GFP moieties on the mother centrioles. Data are presented as Mean $\pm S D$. $N=3$ wing discs, $n \geq 50$ centrioles in total for each protein marker. Statistical significance was assessed using an unpaired t test with Welch's correction (for Gaussian-distributed data) or an unpaired Mann-Whitney test (ns, not significant; ${ }^{* *}, \mathrm{P}<0.001 ;{ }^{* * * *}, \mathrm{P}<0.0001$ ). (C) The position of each indicated GFP marker (coloured solid line) is overlaid on a schematic representation of the Trichonympha EM-tomogram-derived cartwheel 
structure (Guichard et al., 2013). Coloured boxes on the schematic illustration indicate the likely ranges $( \pm 1 S D)$ for the average position of the GFP-moiety on each fusion-protein. Note that the data for Sas-6-GFP and Sas-4-GFP were acquired on the same microscope set-up, but were analysed by singlemolecule localization microscopy and were shown previously (Gartenmann et al., 2017). They are re-plotted here to indicate the positions of CP110 and Cep97 relative to the outer cartwheel spokes (Sas-6-GFP) and the area linking the cartwheel to the centriole MTs (Sas-4-GFP). Cep97 and GFP appear to co-localise with the predicted position of the centriole MTs. 


\section{Figure 2}

A

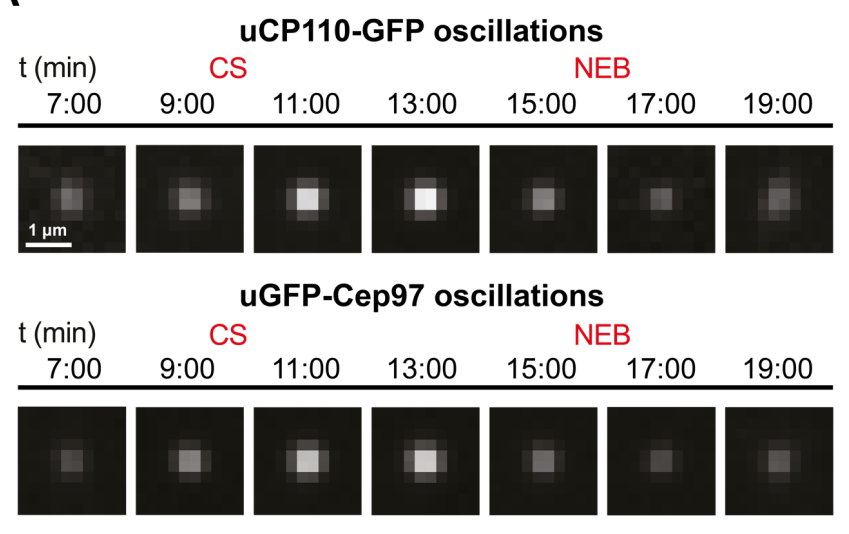

C

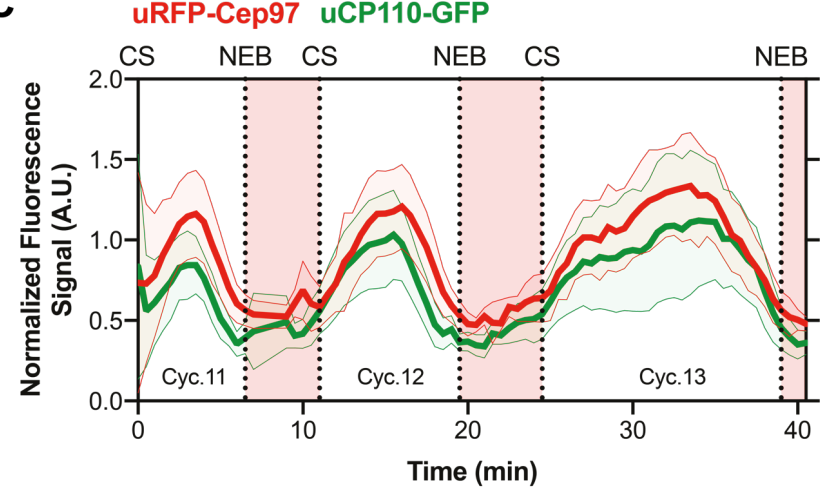

B CS: Centrosome Separation NEB: Nuclear Envelope Breakdown $=\begin{gathered}\text { uCP110-GFP or uGFP-Cep97 } \\ \text { (Mean } \pm \text { SD) }\end{gathered} \quad \vdots \quad \vdots$ Mitosis UCP110-GFP

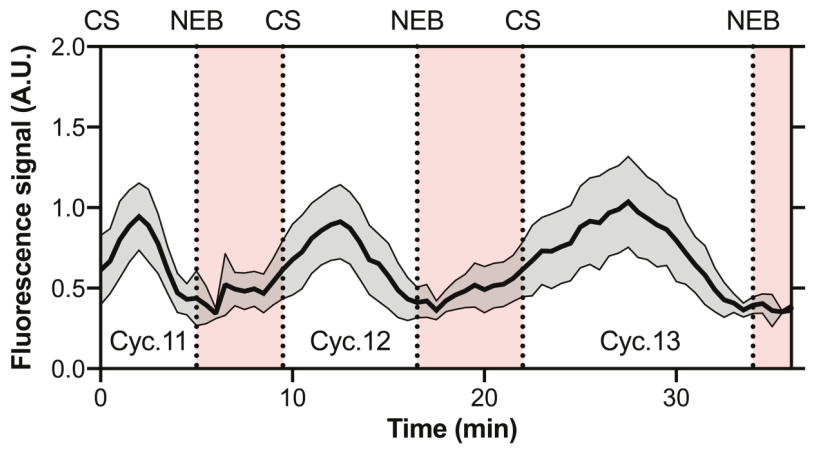

uGFP-Cep97

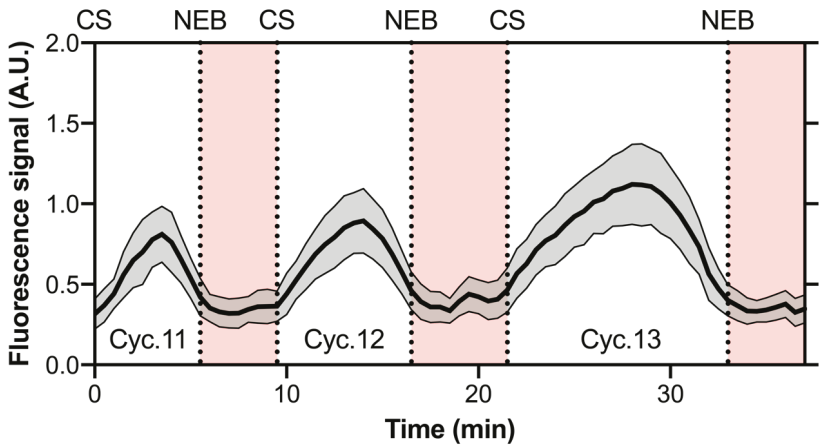

Figure 2. CP110 and Cep97 are recruited to the distal-end of centrioles in oscillations.

(A) Micrographs from two different embryos illustrate the recruitment of uCP110-GFP and uGFP-Cep97 to centrioles over time during nuclear cycle 12 - obtained by superimposing all the uCP110-GFP $(n=150)$ or uGFP-Cep97

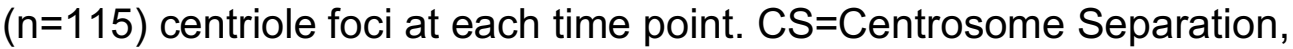
NEB=Nuclear Envelope Breakdown. (B) Quantification of the centrosomal fluorescence levels of uCP110-GFP or UGFP-Cep97 in individual embryos during cycles 11-13. The graphs are representative of 4 independent embryos expressing either UCP110-GFP or UGFP-Cep97 with $n=66$ or 51 centrioles per embryo (on average), respectively. (C) Quantification of the centrosomal fluorescence levels of uCP110-GFP and URFP-Cep97 in an individual embryo during cycles 11-13. The graph is representative of 6 independent embryos with $n=71$ centrioles per embryo (on average). 
A

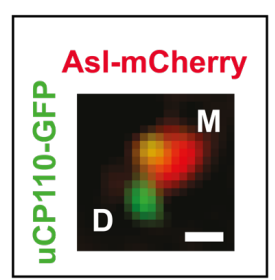

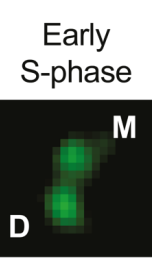

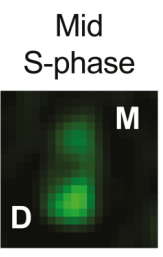

\section{Figure 3}

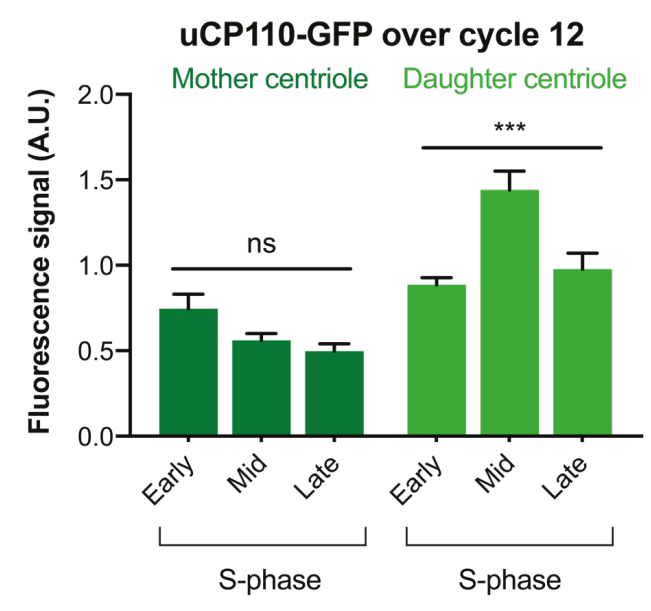

C uCP110-GFP over cycles 12 and 13

$\rightarrow$ Daughter centriole $\quad \bullet$ Mother centriole

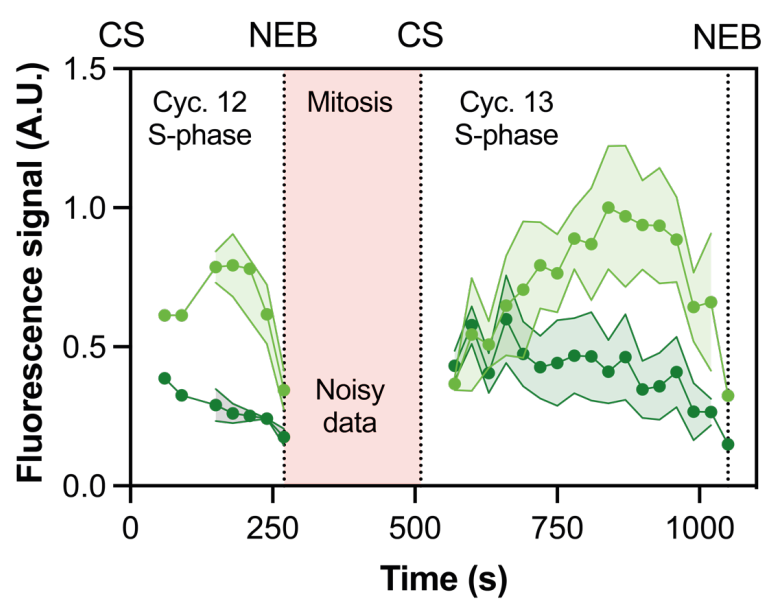

B
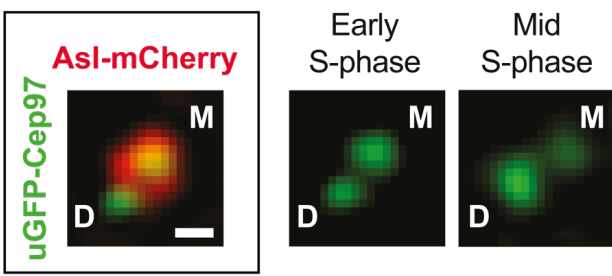

Late S-phase
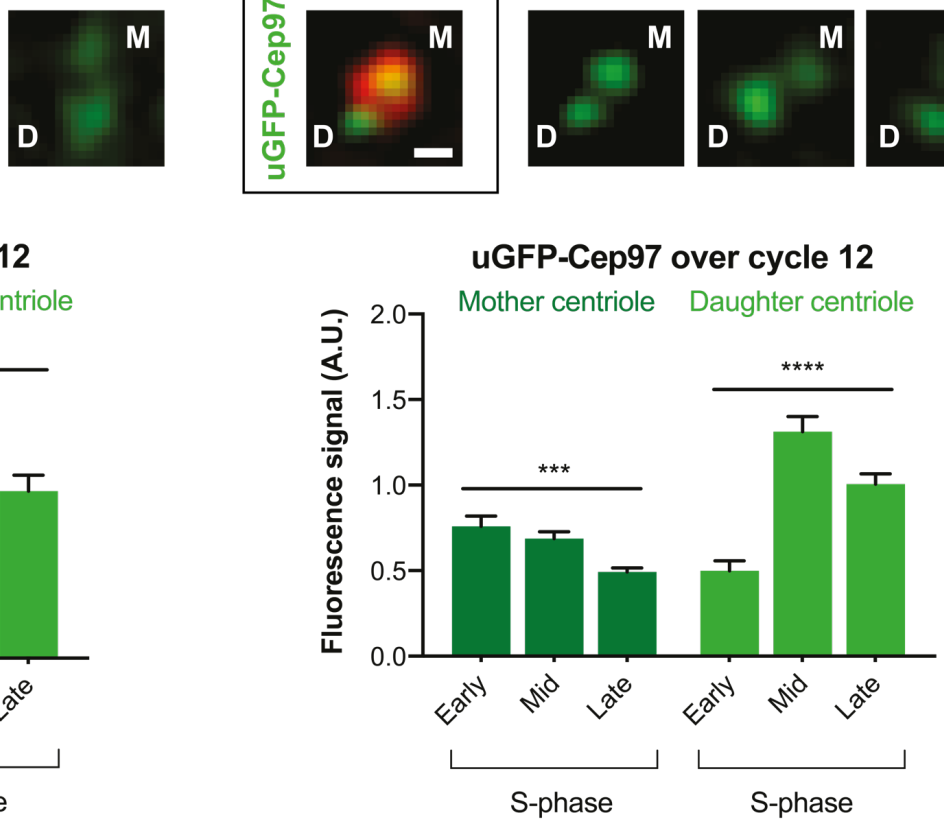

D uGFP-Cep97 over cycle 12 and 13

$\rightarrow$ Daughter centriole $\rightarrow$ Mother centriole

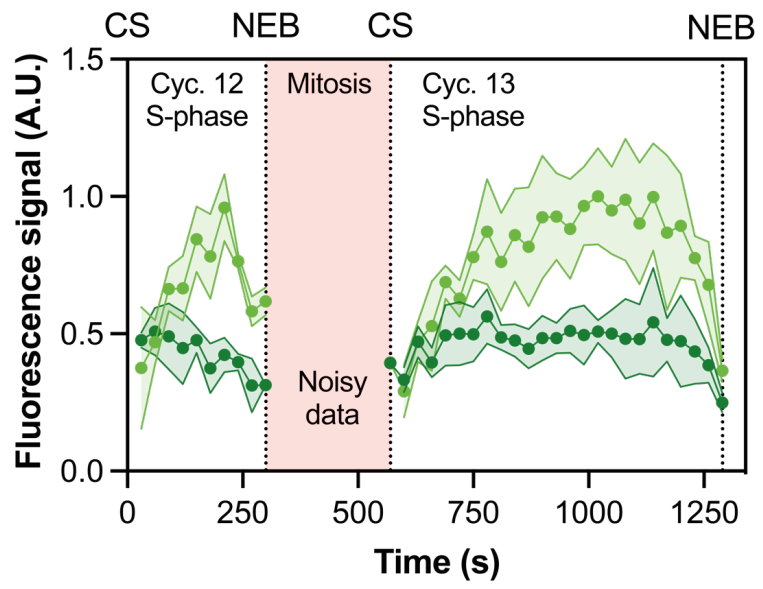

Figure 3. Centriolar levels of CP110-GFP and GFP-Cep97 oscillate exclusively on the growing daughter centriole.

(A and B) Airy-scan micrographs of centrioles at the indicated stages of Sphase in embryos that express Asl-mCherry and (A) uCP110-GFP or (B) uGFP-Cep97; $D=$ daughter centriole; $M=$ mother centriole; Scale bars $=0.2 \mu \mathrm{m}$. Bar charts quantify the centriolar levels of (A) uCP110-GFP or (B) uGFPCep97 on the mother (red bars) and daughter (green bars) centrioles at various time points in S-phase. Note that the levels of uCP110-GFP and uGFP-Cep97 on the mother centrioles do not oscillate and, if anything, appear to gradually decline instead. Data are presented as Mean \pm SEM. $N \geq 7$ 
embryos. For uGFP-Cep97, n=1-14 centrioles per embryo. For uCP110-GFP, $n=1-9$ centrioles per embryo. Statistical significance was assessed using an ordinary one-way ANOVA test (for Gaussian-distributed data) or a KruskalWallis test (ns, not significant; $\left.{ }^{* * *}, \mathrm{P}<0.001 ;{ }^{* * *}, \mathrm{P}<0.0001\right)$. (C and D) Graphs quantify the fluorescence intensity of (C) uCP110-GFP or (D) UGFPCep97 on mother (dark green) and daughter (light green) centrioles in individual embryos over Cycles 12-13 (with data acquired using Airy-scan microscopy). The fluorescence signal was too low in mitosis and early Sphase for reliable quantification (denoted by "Noisy data). CS = Centrosome Separation, NEB = Nuclear Envelope Breakdown. Data are presented as Mean \pm SD. For uGFP-Cep97, $n=1-4$ or 1-10 daughter and mother centrioles per time point in Cycles 12 and 13, respectively. For uCP110-GFP, $n=1-3$ or 1-8 daughter and mother centrioles per time point in Cycles 12 and 13, respectively. $\mathrm{CS}=$ Centrosome Separation, NEB=Nuclear Envelope Breakdown. 
Figure 4
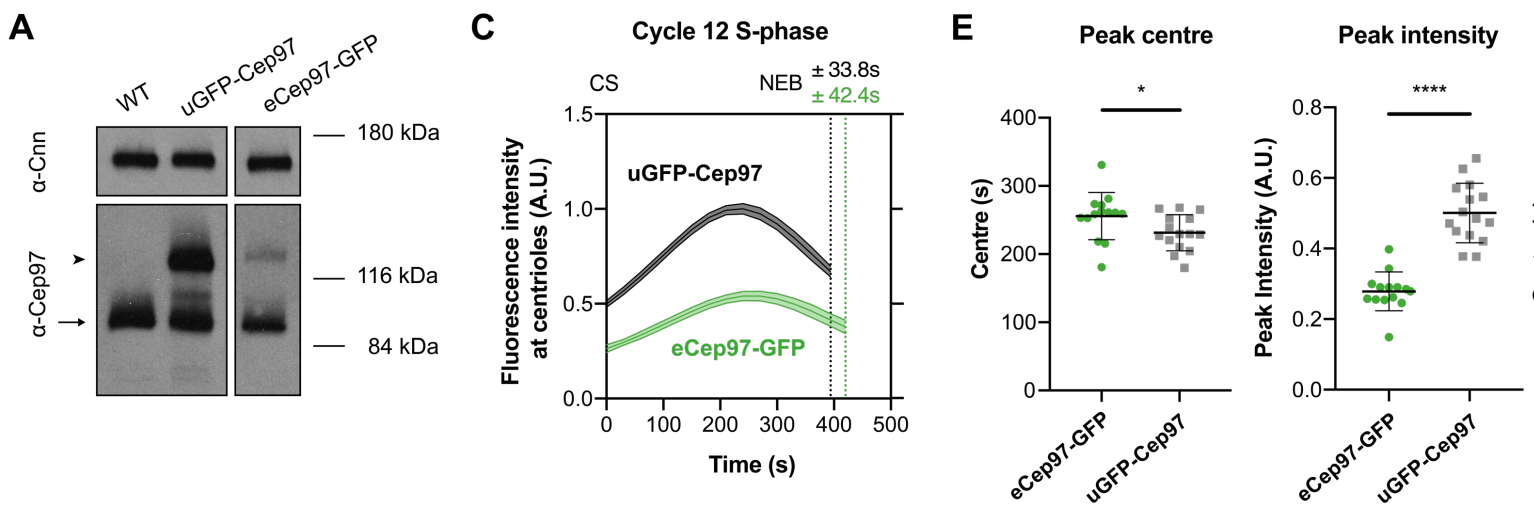

Peak centre normalized
based on S-phase length
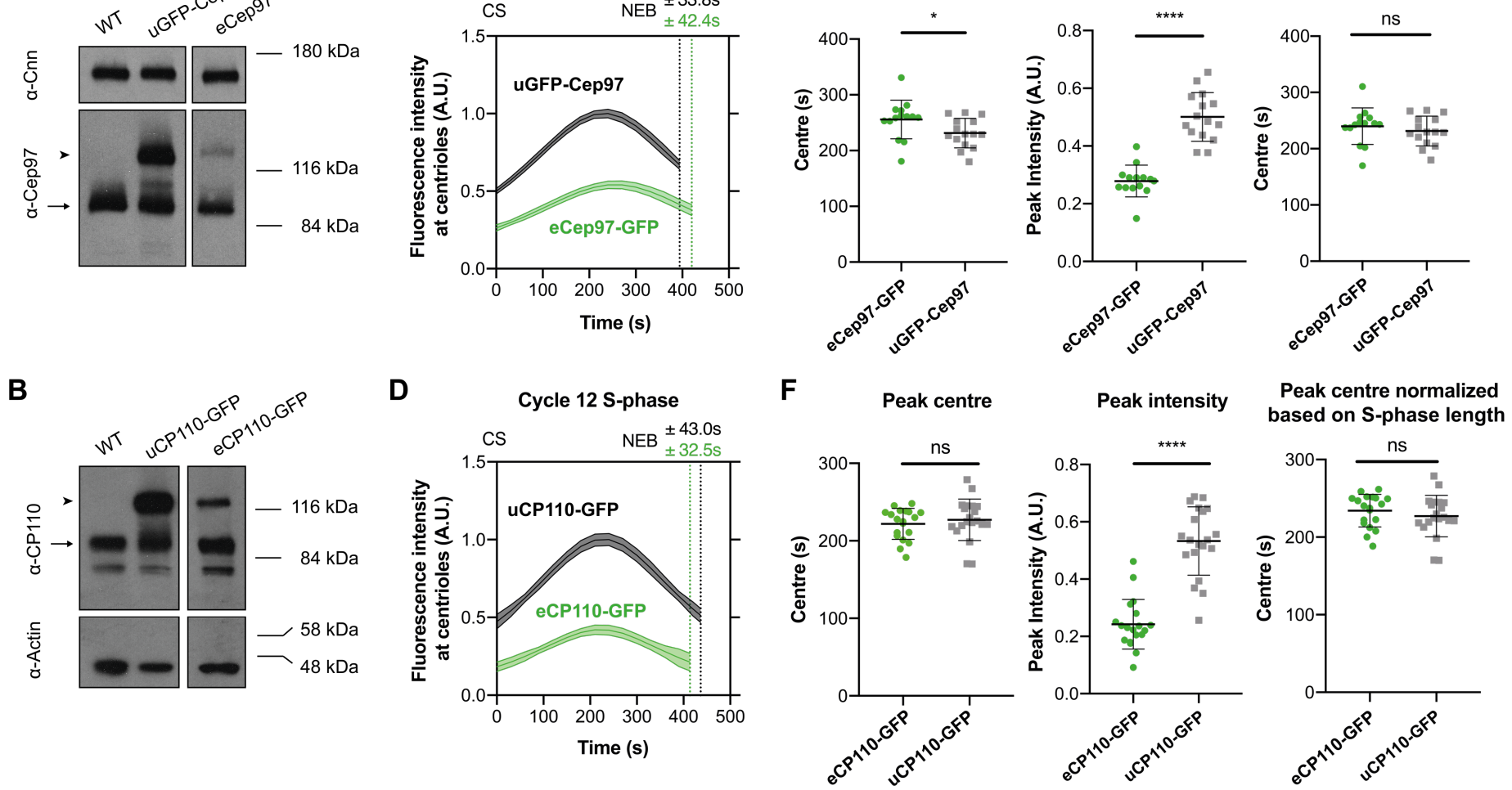

Figure 4. Higher levels of CP110/Cep97 expression promotes faster, and higher levels of, its centriolar recruitment.

(A and B) Western blots comparing Cep97 or CP110 expression levels in embryos expressing one copy of either eCep97-GFP or uGFP-Cep97 (A), or eCP110-GFP or UCP110-GFP (B), as indicated. WT embryos (on left-most lane of each panel) were used to indicate the endogenous level of expression for either protein. The endogenous Cep97 and CP110 bands are indicated with arrows. The bands corresponding to the GFP-tagged proteins are indicated with arrowheads. Actin and $\mathrm{Cnn}$ are shown as loading controls. (C and D) Graphs compare the mean CP110/Cep97-GFP oscillation profiles during nuclear cycle 12 in embryos expressing (C) uGFP-Cep97 or eCep97GFP and (D) UCP110-GFP or eCP110-GFP, as indicated. Data are presented as Mean \pm SEM. (E and F) Bar charts quantify several parameters of the oscillation derived from the profiles shown in ( $C$ and $D)$. Data are presented as Mean $\pm S D$. $N \geq 14$ embryos per group, $n \geq 9$ centrioles per embryo. Statistical significance was assessed using an unpaired t test with Welch's correction (for Gaussian-distributed data) or an unpaired Mann-Whitney test (ns, not significant; $\left.{ }^{*}<0.05 ;{ }^{* * * *}, \mathrm{P}<0.0001\right)$. CS=Centrosome Separation, $\mathrm{NEB}=$ Nuclear Envelope Breakdown. 


\section{Figure 5}

A

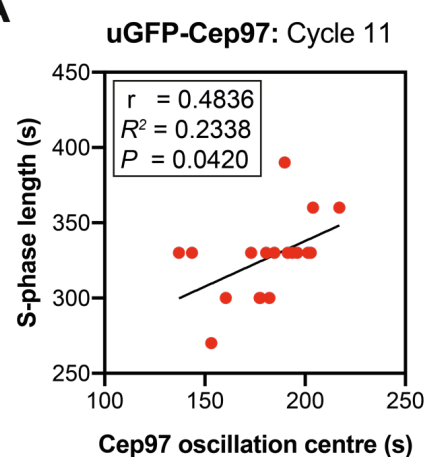

B

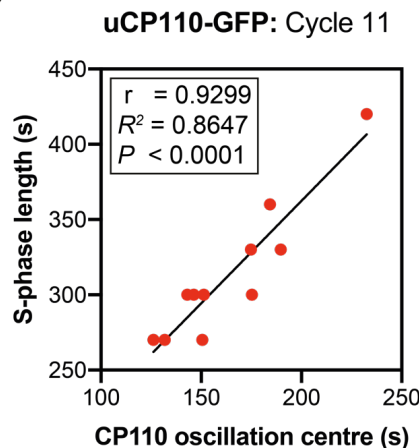

uGFP-Cep97: Cycle 12

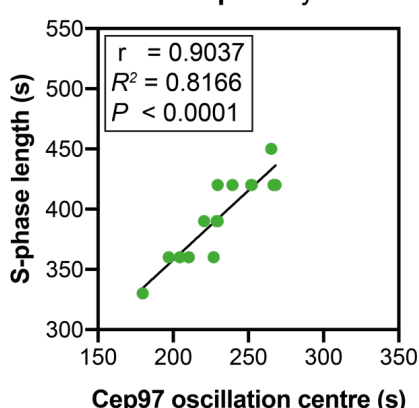

uCP110-GFP: Cycle 12

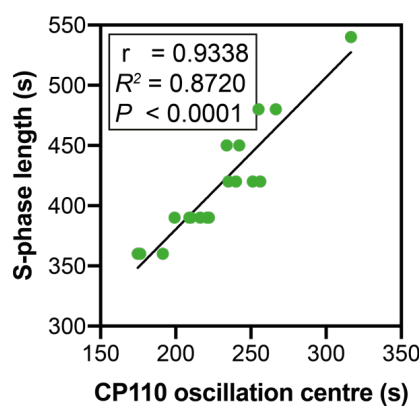

uGFP-Cep97: Cycle 13

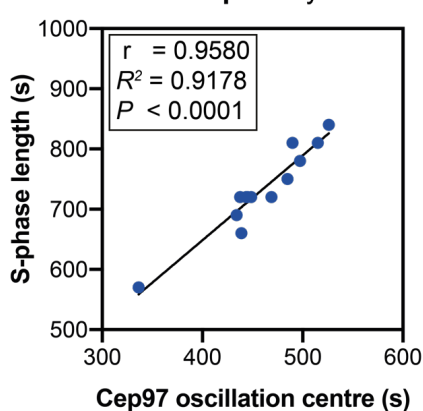

uCP110-GFP: Cycle 13

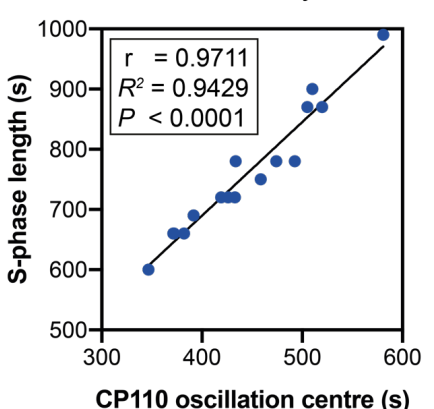

C

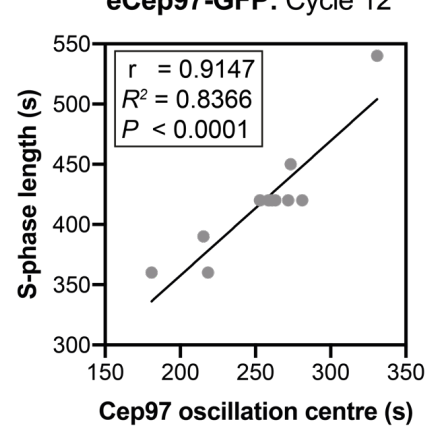

eCP110-GFP: Cycle 12

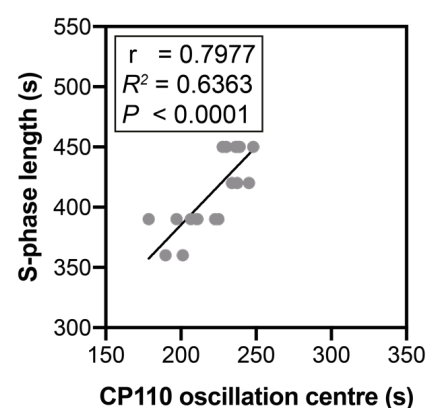

Figure 5. CP110/Cep97 oscillations are strongly correlated with the progression of the cell cycle in fly embryos.

Scatter plots show a positive correlation between S-phase length and the centre (phase) of (A) UGFP-Cep97 and (B) uCP110-GFP oscillations in Cycle 11-13, and of (C) eCep97-GFP and eCP110-GFP oscillations in Cycle 12. The plots were regressed using the line function in GraphPad Prism 8. Correlation strength was examined using Pearson's correlation coefficient $(0.40<r<0.60=$ moderate; $r>0.60=$ strong $)$, and the significance of correlation was determined by the $p$-value $(P<0.05)$. 
A

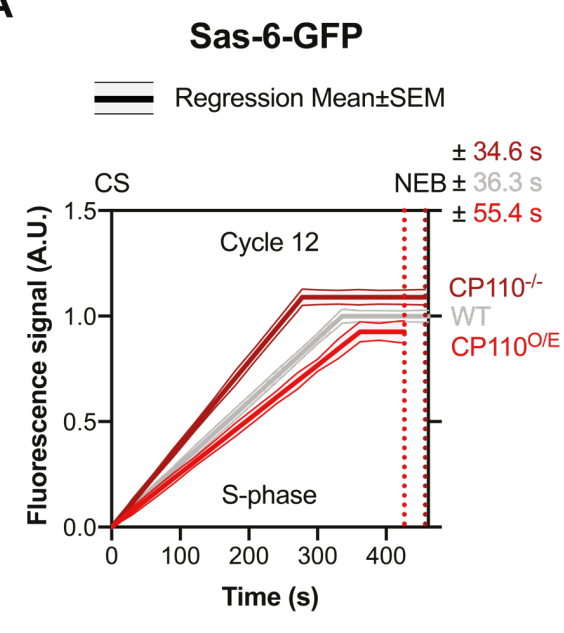

B

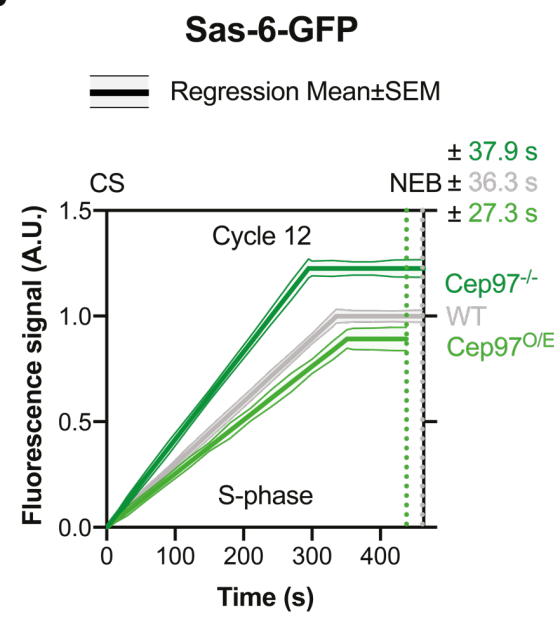

Figure 6

C

Cartwheel growth under various CP110 dosages

Growth rate

Growth period

Intensity acquired
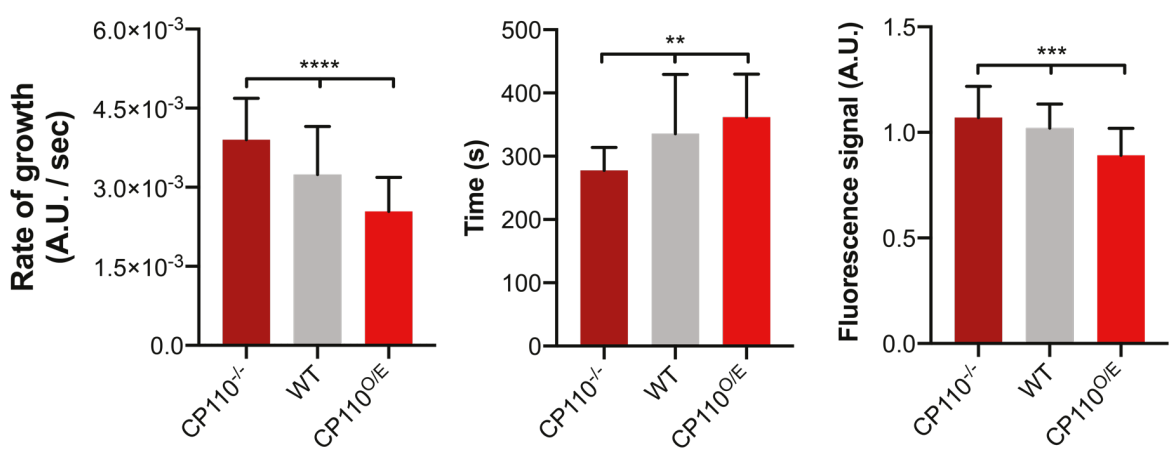

D

Cartwheel growth under various Cep97 dosages
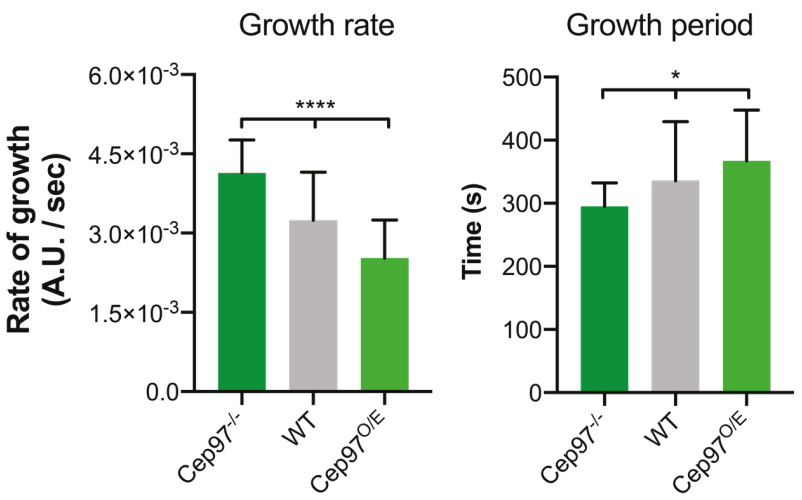

Intensity acquired

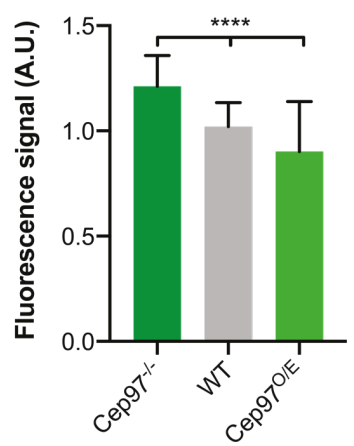

Figure 6. CP110 and Cep97 levels influence the rate and period of centriole cartwheel growth.

(A and B) Graphs compare the mean Sas-6-GFP incorporation profile-as a proxy for centriole cartwheel growth (Aydogan et al., 2018) - during nuclear cycle 12 in WT embryos or in embryos lacking or overexpressing (A) CP110 or (B) Cep97, as indicated. Data are presented as Mean \pm SEM. (C and D) Bar charts quantify several parameters of cartwheel growth derived from the profiles shown in $(A)$ and $(B)$ respectively. Data are presented as Mean $\pm S D$. $\mathrm{N} \geq 14$ embryos per group, $\mathrm{n} \geq 40$ centrioles on average per embryo. Statistical significance was assessed using an ordinary one-way ANOVA test (for Gaussian-distributed data) or a Kruskal-Wallis test $\left({ }^{*}, \mathrm{P}<0.05 ;{ }^{* *}, \mathrm{P}<0.01\right.$; ${ }^{* * *}$, $\left.\mathrm{P}<0.001 ;{ }^{* * * *}, \mathrm{P}<0.0001\right)$. 


\section{Figure 7}

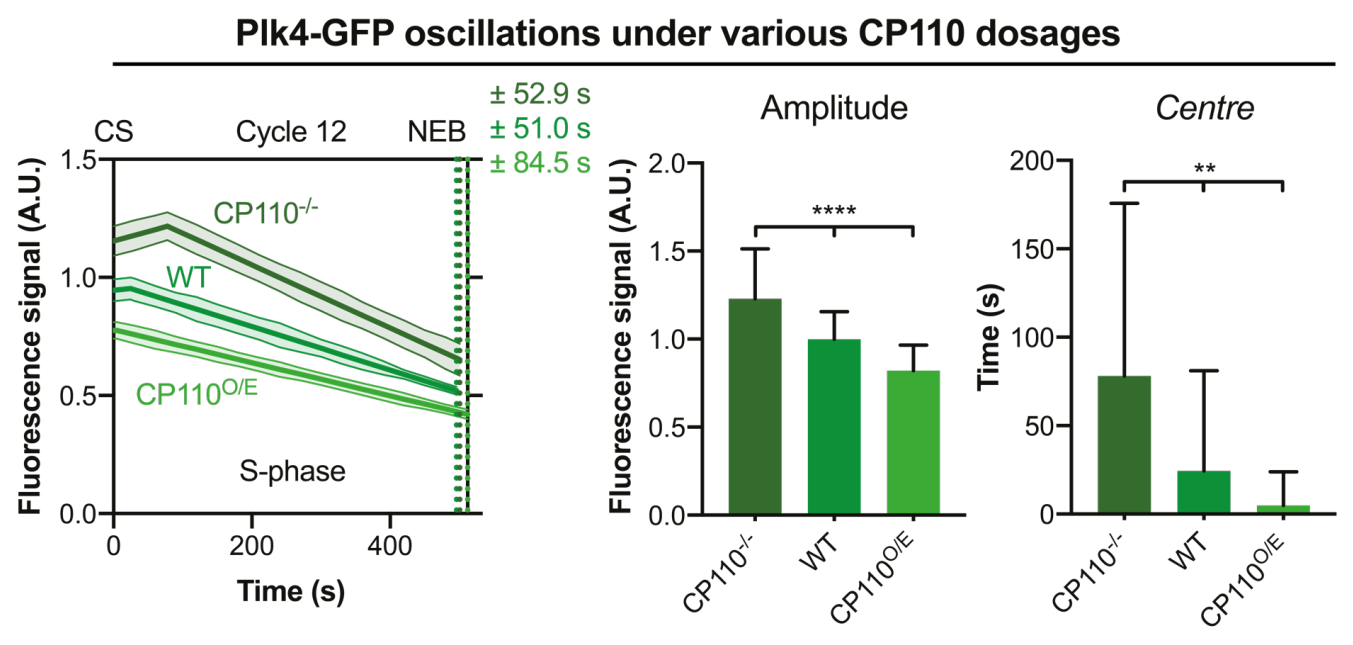

PIk4-GFP oscillations under various Cep97 dosages
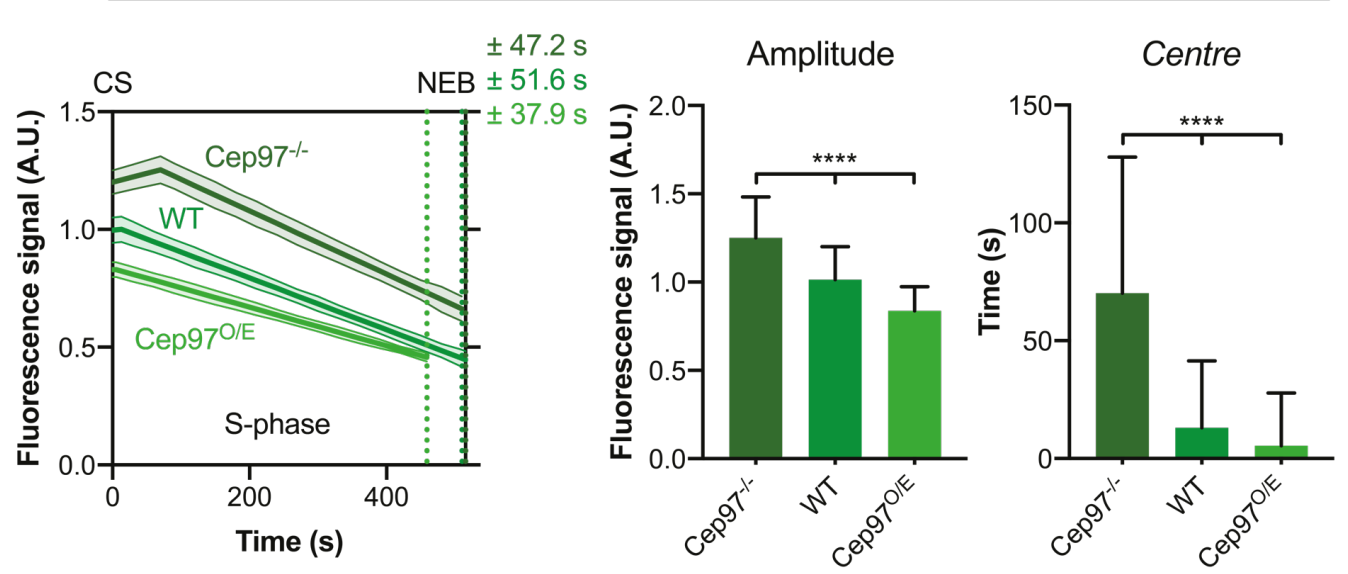

Figure 7. CP110 and Cep97 levels influence the parameters of the PIk4 oscillation.

Graphs show discrete Plk4-GFP oscillations in S-phase during nuclear cycle 12 in embryos expressing various levels of either CP110 or Cep97. Data are presented as Mean \pm SEM. The Plk4 oscillation was previously shown to control the parameters of centriole growth (Aydogan et al., 2020).

Corresponding bar charts therefore compare the amplitude and centre of the discrete PIk4-GFP oscillation under the indicated conditions. Data are presented as Mean $\pm S D$. $N \geq 16$ embryos per group, $n \geq 45$ centriole pairs per embryo. Statistical significance was assessed using an ordinary one-way ANOVA test (for Gaussian-distributed data) or a Kruskal-Wallis test (**, $\left.\mathrm{P}<0.01 ;{ }^{* * * *}, \mathrm{P}<0.0001\right)$. 


\section{Figure 8}

A

Peak Counting Spectroscopy (PeCoS)

Cytoplasmic PIk4-GFP

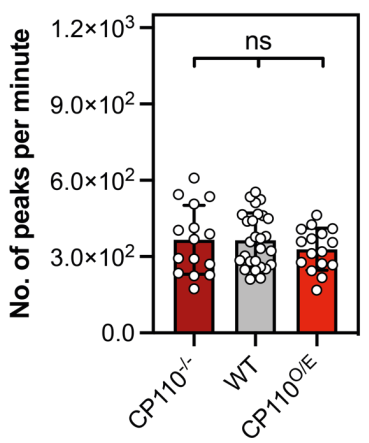

C

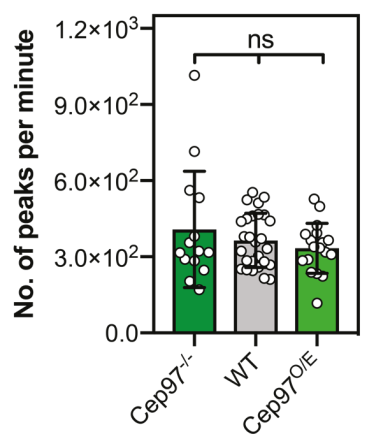

B

Fluorescence Correlation Spectroscopy (FCS)

Cytoplasmic uCP110-GFP Cytoplasmic uGFP-Cep97
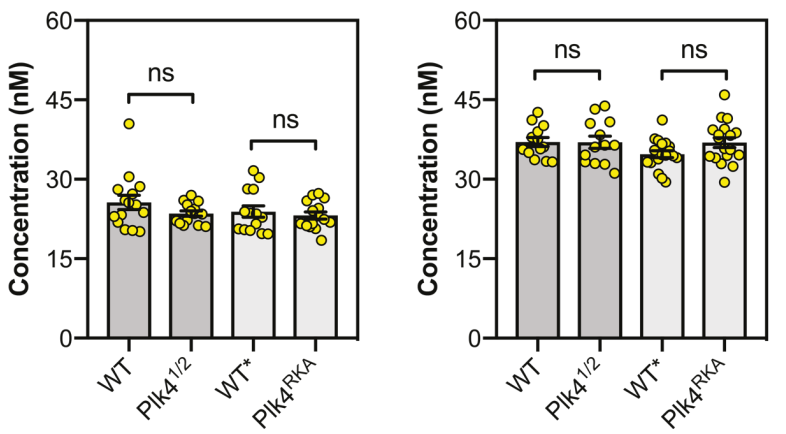

Western Blot Analysis

Cytoplasmic CP110

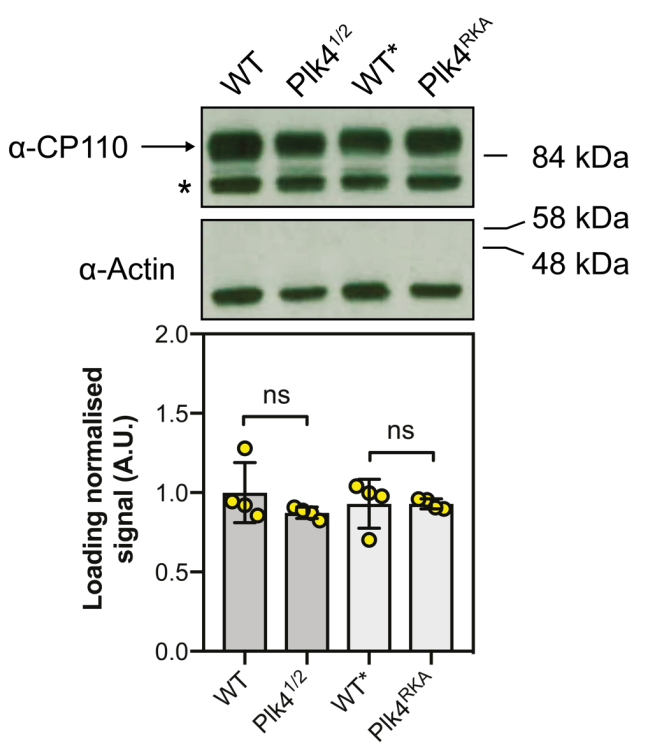

Cytoplasmic Cep97
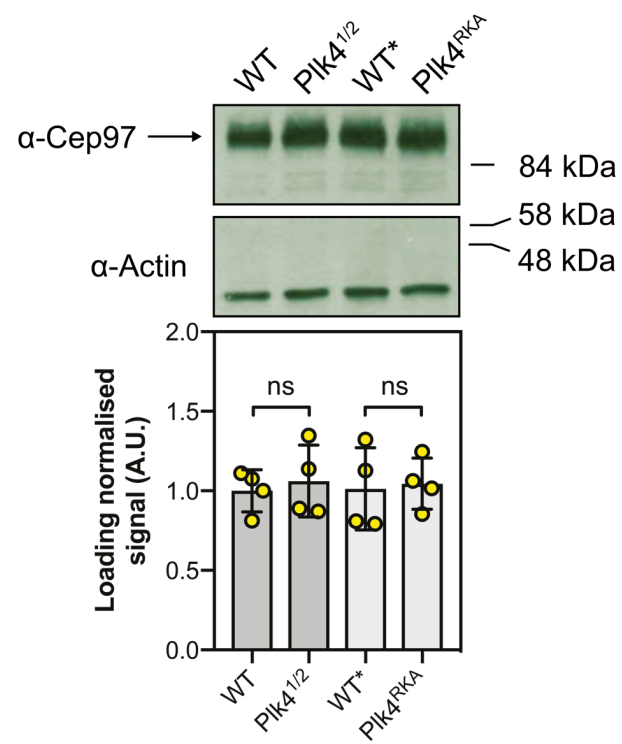

Figure 8. Altering the cytoplasmic levels or activity of Plk4 does not detectably alter the cytoplasmic levels of CP110 or Cep97 and vice versa.

(A) Graphs comparing the Auto Correlation Functions (ACFs) of uCP110-GFP and UGFP-Cep97 in either (i) WT versus Plk4 ${ }^{1 / 2}$ embryos or (ii) embryos expressing an additional copy of endogenous Plk4 (WT*) versus embryos expressing an additional copy of endogenous Plk4 carrying a previously described mutation that reduces its kinase activity (Plk4 ${ }^{\text {RKA }}$ ) (Aydogan et al., 2018). Data are presented as Mean \pm SEM. (iii) Pie charts show the results of the model fitting based on the Bayesian Information Criterion. For both uCP110-GFP (left) and uGFP-Cep97 (right), the diffusion behaviour was best described by Model 4, which consists of one diffusing species with one 
blinking and one triplet state of the fluorophore. (iv) Scatter plots show all the outliers (red dots) and the count-rate per molecule (CPM) of each $10 \mathrm{sec}$ FCS recording as a function of its background-corrected cytoplasmic concentration. Data points below this cut-off were excluded from further analyses-see (Aydogan et al., 2020) for a justification of this cut-off. (B) Bar charts quantify the background-corrected FCS measurements of UCP110-GFP or uGFPCep97 under indicated conditions from (Ai) and (Aii). Data are presented as Mean \pm SEM. Every data point represents the average of 4-6 recordings from each embryo measured. Statistical significance was assessed using an ordinary unpaired t-test (for Gaussian-distributed data) or a Mann-Whitney test (ns, not significant). (C) (Upper panel) Western blots show the cytoplasmic expression of endogenous CP110 and Cep97 (arrows) under the same conditions used to measure the concentration of UCP110-GFP and uGFP-Cep97 by FCS in (A and B). Actin is shown as a loading control, and prominent non-specific bands are indicated $\left(^{*}\right)$. Representative blots are shown from four technical repeats. (Lower panel) Bar charts quantify the loading-normalised levels of CP110 and Cep97 from the four technical repeats. Data are presented as Mean $\pm S D$. Statistical significance was assessed using a Mann-Whitney test (ns, not significant). See Materials and Methods for further details on the blot quantification protocol. (D) Bar charts quantify PeCoS (Aydogan et al., 2020) measurements of Plk4-GFP in embryos expressing various levels of CP110 and Cep97, as indicated. Every data point represents $1 \times 180 \mathrm{sec}$ measurement from an individual embryo. Data are presented as Mean $\pm S D$. Statistical significance was assessed using an ordinary one-way ANOVA test (for Gaussian-distributed data) or a KruskalWallis test (ns, not significant). 


\section{Supplementary figures and legends}

\section{Figure S1}

A

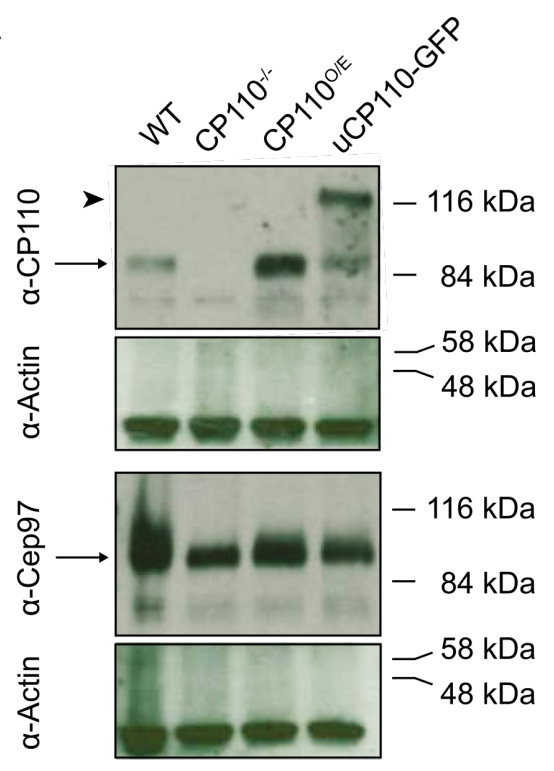

B

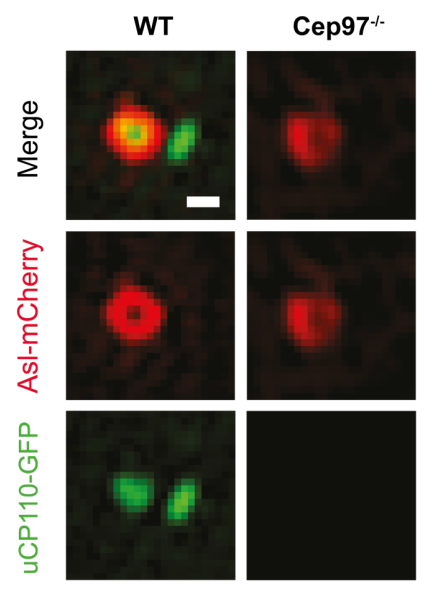

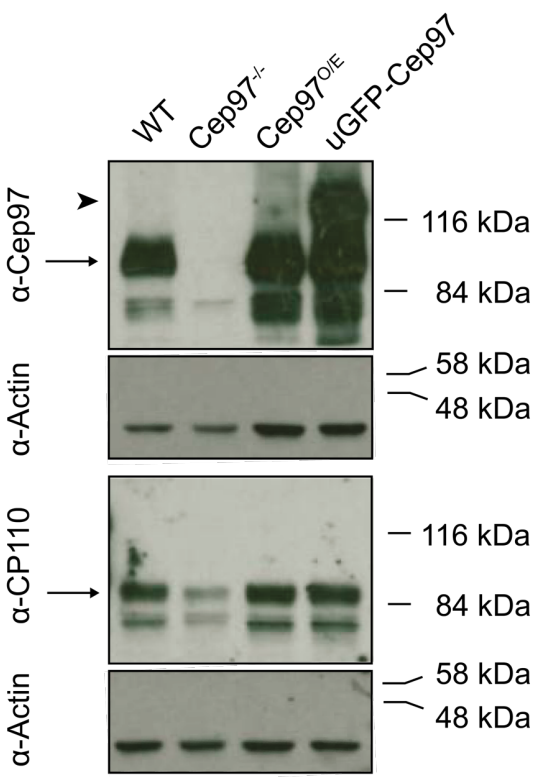

C

D
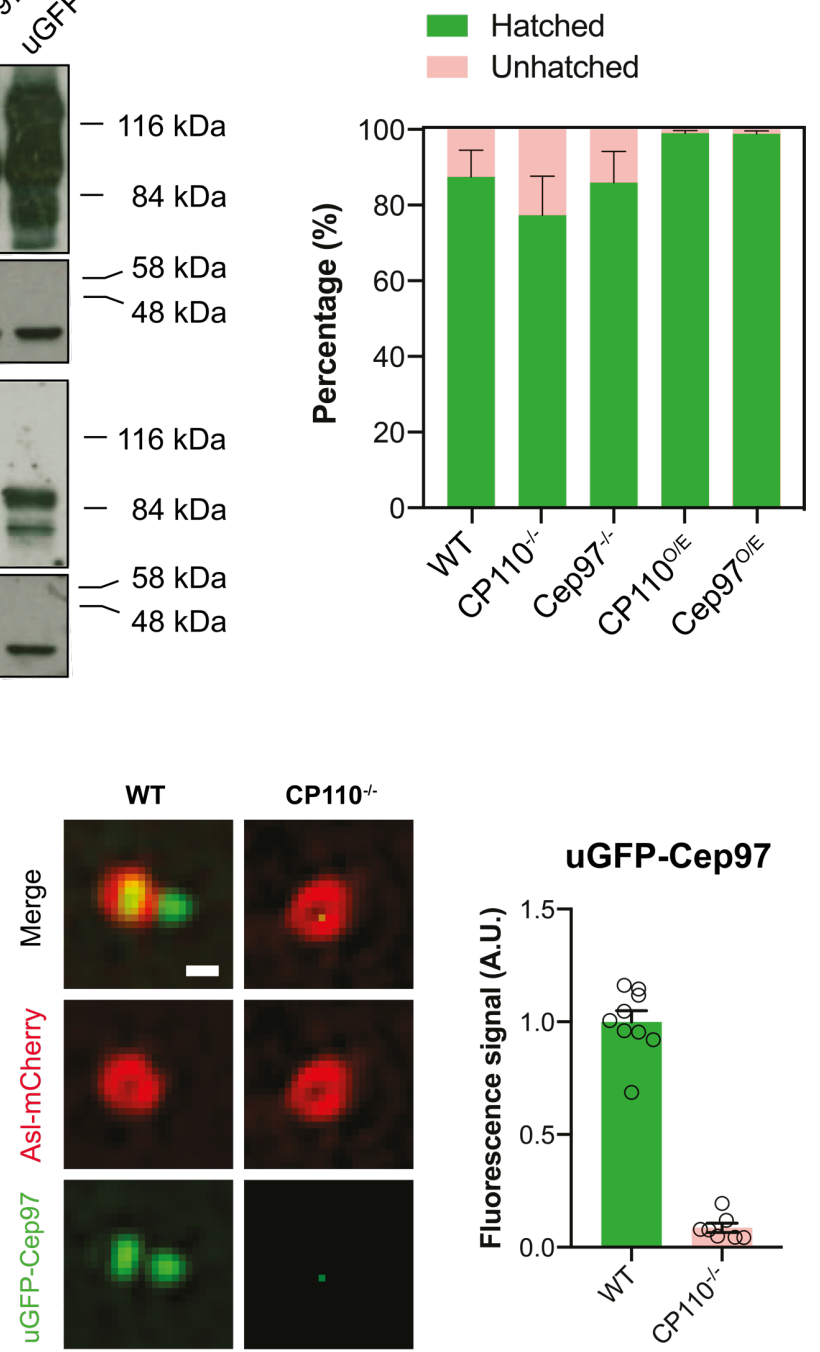

Figure S1. CP110 and Cep97 are co-dependent for their centriolar localisation and partially co-dependent for their cytoplasmic stability. (A) Western blots show the protein levels of CP110 and Cep97 in embryos of various genotypes, as indicated. Actin is shown as a loading control.

Representative blots are shown from three technical repeats. Note that there appears to be slightly less Cep97, which is less smeared, in the absence of CP110, while there is clearly less CP110 in the absence of Cep97. (B and C) Airy-scan micrographs shows the centriole localisation of either (B) UCP110GFP in WT and Cep97 $7^{-/-}$embryos or (C) UGFP-Cep97 in WT and CP110-embryos; Asl-mCherry labels the mother centrioles (Scale bar=0.2 $\mu \mathrm{m}$ ). Bar charts quantify the centriolar levels of uCP110-GFP or uGFP-Cep97, as indicated. For this quantification, Asl-mCherry was used as a centriole marker 
to select the ten brightest centriole pairs in each embryo 20 minutes into Sphase of Cycle 14 (when the signal should be the brightest). Data are presented as Mean \pm SEM. $N \geq 7$ embryos, $n=10$ centrioles per embryo. (D) Bar chart indicates the embryo hatching frequency in wild type flies (Oregon $R$ ) or in flies of the indicated genotypes. 
A

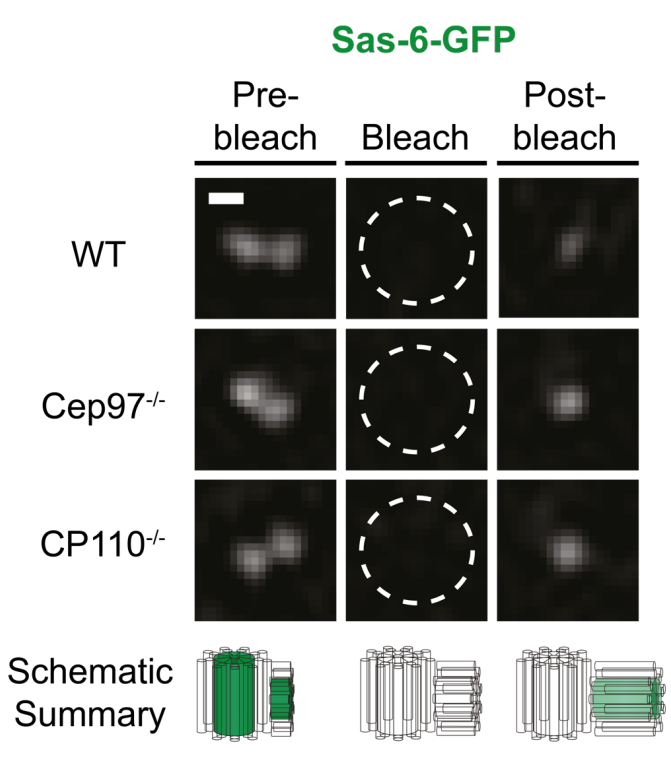

C

Wild-type

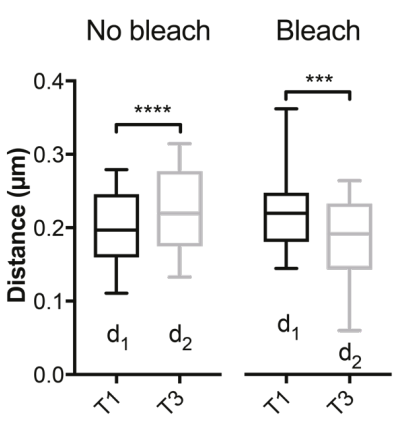

\section{Figure S2}

B

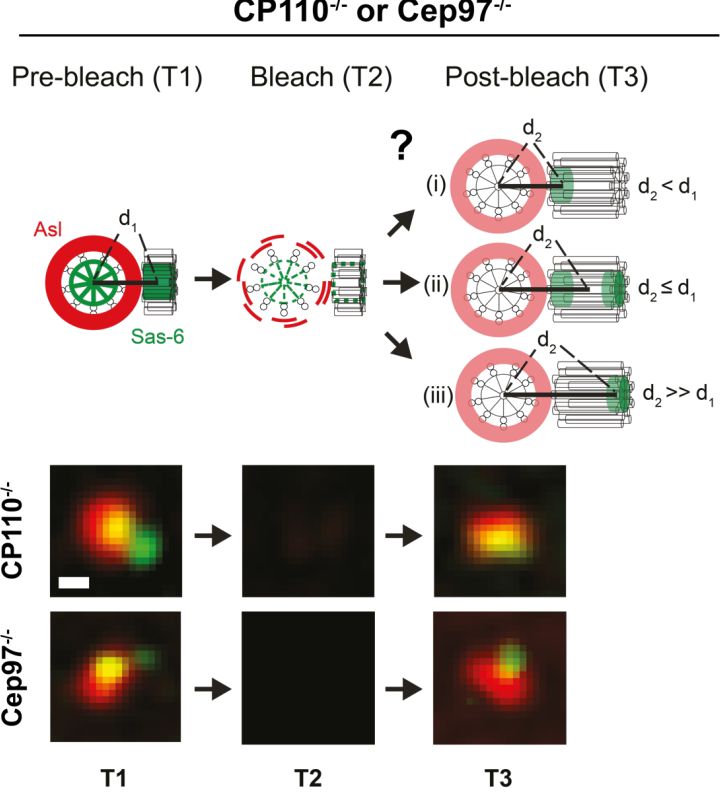

D

Wild-type

Cep97-

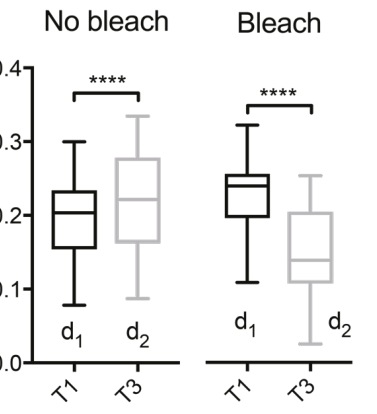

Figure S2. The centriole cartwheel continues to grow exclusively from the proximal-end of the daughter centriole in CP110 ${ }^{-/-}$and Cep97-Iembryos.

(A) Micrographs show a 3D-SIM-FRAP analysis of Sas-6-GFP dynamics in WT, CP110 $10^{-/-}$and Cep97 $7^{-/}$embryos. For each condition, a 3D-SIM image of a centriole pair was acquired in early/mid S-phase (Pre-bleach). The centrioles were subsequently photobleached (Bleach), and a 3D-SIM image was acquired $1 \mathrm{~min}$ after photobleaching (Post-bleach). These observations demonstrate that Sas-6-GFP continues to be incorporated exclusively into the growing daughter centriole even in the absence of CP110 or Cep97. Scale bar $=0.2 \mu \mathrm{m} . \mathrm{N} \geq 8$ embryos per group, $\mathrm{n}=3$ centriole pairs on average per embryo. Schematics below each micrograph illustrate our interpretation of the FRAP experiments. (B) Schematics illustrate the assay (Aydogan et al., 2018) that revealed that Sas-6-GFP incorporates into the proximal-end of growing daughter cartwheels (outcome [i]). We used the same assay to test whether this was also the case in CP110/- and Cep97/- embryos. (Lower panel) Airyscan super resolution micrographs show representative centriole images 
during pre-bleach (T1), bleach (T2) and post-bleach (T3) stages of the FRAP experiment in CP110-/- and Cep97 $7^{-/-}$embryos simultaneously expressing AslmCherry and Sas-6-GFP. Scale bar $=0.2 \mu \mathrm{m}$. (C and D) The box and whisker plots show the pre- and post-bleach distance ( $d_{1}$ and $d_{2}$, respectively) between Asl-mCherry on the mother centriole and the newly incorporating Sas-6-GFP on the growing daughter centriole in (C) CP110 or (D) Cep97-/embryos compared to WT controls. In the No bleach control experiment, $\mathrm{d}_{2}>$ $\mathrm{d}_{1}$ for all conditions, reflecting the growth of the daughter centriole between T1 and T3. In the Bleach experiment, $\mathrm{d}_{2}<<\mathrm{d}_{1}$ for all conditions, indicating that Sas-6-GFP continues to incorporate only into the proximal-end of the centrioles in the absence of CP110 or Cep97. $\mathrm{N} \geq 11$ embryos per condition; $\mathrm{n} \geq 16$ centriole pairs for No Bleach and Bleach groups each. Midlines represent the median, whiskers (error bars) mark the minimum to maximum, and bottom/top of the boxes indicate the first/third quartile of the distribution, respectively. Statistical significance was assessed using a paired $t$ test $\left({ }^{*}\right.$, $\left.\mathrm{P}<0.05 ;{ }^{* *}, \mathrm{P}<0.01 ;{ }^{* * *}, \mathrm{P}<0.001 ;{ }^{* * * *}, \mathrm{P}<0.0001\right)$. 


\section{Supplementary videos and captions}

Movie S1. Monitoring uCP110-GFP oscillations in a Drosophila embryo. Time-lapse video of an embryo expressing uCP110-GFP, observed on a spinning-disk confocal microscope through nuclear cycles 11-13. The movie is a maximum-intensity projection that has been photo-bleach corrected, but not background subtracted for visual clarity. Time (min:sec) is shown at the top left, and the developmental stage of the embryo is indicated at the bottom left.

\section{Movie S2. Monitoring uGFP-Cep97 oscillations in a Drosophila embryo.} Time-lapse video of an embryo expressing uGFP-Cep97, observed on a spinning-disk confocal microscope through nuclear cycles 11-13. The movie is a maximum-intensity projection that has been photo-bleach corrected, but not background subtracted for visual clarity. Time (min:sec) is shown at the top left, and the developmental stage of the embryo is indicated at the bottom left.

Movie S3. Monitoring uCP110-GFP and uRFP-Cep97 oscillations simultaneously in the same embryo.

Time-lapse movie of an embryo expressing uCP110-GFP and uRFP-Cep97, observed on a spinning-disk confocal microscope through nuclear cycles 1113. The movie is a maximum-intensity projection that has been photo-bleach corrected, but not background subtracted for visual clarity. Time (Min:Sec) is shown at the top left, and the developmental stage of the embryo is indicated at the bottom left. 